\title{
Revascularization of ischemic tissues by PlGF treatment, and inhibition of tumor angiogenesis, arthritis and atherosclerosis by anti-Flt1
}

\author{
Aernout Luttun ${ }^{1}$, Marc Tjwa ${ }^{1}$, Lieve Moons ${ }^{1}$, Yan Wu$^{4}$, Anne Angelillo-Scherrer ${ }^{5}$, \\ Fang LiaO ${ }^{4}$, Janice A. NagY ${ }^{6}$, Andrea Hooper ${ }^{4}$, Josef Priller ${ }^{7}$, Bert De Klerck ${ }^{2}$, \\ Veerle Compernolle ${ }^{1}$, Evis Daci ${ }^{3}$, Peter Bohlen ${ }^{4}$, Mieke Dewerchin ${ }^{1}$, \\ Jean-Marc Herbert ${ }^{8}$, Roy Fava ${ }^{9}$, Patrick Matthys ${ }^{2}$, Geert Carmeliet ${ }^{3}$, Désiré Collen ${ }^{1}$, \\ Harold F. Dvorak ${ }^{6}$, Daniel J. Hicklin ${ }^{4} \&$ Peter Carmeliet $^{1}$ \\ ${ }^{1}$ Center for Transgene Technology and Gene Therapy, Flanders Interuniversity Institute for Biotechnology; \\ ${ }^{2}$ Laboratory of Immunobiology, Rega Institute for Medical Research; ${ }^{3}$ Laboratory of Experimental Medicine and \\ Endocrinology, University of Leuven, Leuven, Belgium \\ ${ }^{4}$ ImClone Systems Inc., New York, New York, USA \\ ${ }^{5}$ Division of Angiology and Hemostasis, University Medical Center, Geneva, Switzerland \\ ${ }^{6}$ Department of Pathology, Beth Israel Deaconess Medical Center, \\ Harvard Medical School, Boston, Massachusetts, USA \\ ${ }^{7}$ Department of Neurology, Charité, Humboldt-University, Berlin, Germany \\ ${ }^{8}$ Cardiovascular/Thrombosis Research Department, Sanofi-Synthélabo, Toulouse Cedex, France \\ ${ }^{9}$ Department of Veterans Affairs Medical Center, White River Junction, Vermont and Department of Medicine, \\ Dartmouth Medical School, Hanover, New Hampshire, USA \\ A.L. and M.T. contributed equally to this study. \\ Correspondence should be addressed to P.C.; email: peter.carmeliet@med.kuleuven.ac.be
}

Published online: 1 July 2002, doi:10.1038/nm731

\begin{abstract}
The therapeutic potential of placental growth factor (PIGF) and its receptor Flt 1 in angiogenesis is poorly understood. Here, we report that PIGF stimulated angiogenesis and collateral growth in ischemic heart and limb with at least a comparable efficiency to vascular endothelial growth factor (VEGF). An antibody against Flt1 suppressed neovascularization in tumors and ischemic retina, and angiogenesis and inflammatory joint destruction in autoimmune arthritis. Anti-Flt1 also reduced atherosclerotic plaque growth and vulnerability, but the atheroprotective effect was not attributable to reduced plaque neovascularization. Inhibition of VEGF receptor Flk1 did not affect arthritis or atherosclerosis, indicating that inhibition of Flk1-driven angiogenesis alone was not sufficient to halt disease progression. The anti-inflammatory effects of anti-Flt 1 were attributable to reduced mobilization of bone marrow-derived myeloid progenitors into the peripheral blood; impaired infiltration of Flt1-expressing leukocytes in inflamed tissues; and defective activation of myeloid cells. Thus, PIGF and Flt 1 constitute potential candidates for therapeutic modulation of angiogenesis and inflammation.
\end{abstract}

Vascular endothelial growth factor (VEGF) and its receptor tyrosine kinase-2 (VEGFR-2 or Flk1/KDR) have received much attention for therapeutic stimulation or inhibition of angiogenesis ${ }^{1}$. However, VEGF induces only transient and modest improvement of cardiac function in some, but not in all patients with ischemic heart disease ${ }^{2,3}$, suggesting that additional angiogenic agents may be required. Inhibitors of VEGF and its receptors are currently evaluated for treatment of cancer and other disorders ${ }^{4}$. Although VEGF binds both receptor tyrosine kinases Flk1 and Flt1 (VEGFR-1), inhibitors have been primarily targeted to neutralization of Flk1-activity, based on the assumption that VEGFdriven angiogenesis is primarily mediated via Flk1 (refs. 1,5).

The role of Flt1, to which not only VEGF, but also its homologs placental growth factor (PlGF) and VEGF-B bind, has remained enigmatic, primarily because Flt1 has low tyrosine kinase activity ${ }^{6,7}$ and its signaling pathways remain poorly characterized $^{8}$. Mouse embryos lacking Flt1 succumb due to vascular defects, whereas mice expressing Flt 1 lacking the tyrosine kinase domain survive ${ }^{6,9}$, suggesting that, during development, Flt1 primarily functions as a non-signaling 'reservoir' for VEGF. By displacing VEGF from Flt1, PlGF provides additional VEGF to induce Flk1-mediated angiogenic signaling ${ }^{10}$. However, recent gene-targeting studies indicate that Flt1-mediated signaling may have a significant role in pathological angiogenesis. Indeed, loss of PIGF impairs angiogenesis in ischemic retina, limb and heart, in wounded skin and in cancer, without affecting physiological angiogenesis ${ }^{11}$. Furthermore, genetic truncation of the Flt 1 tyrosine kinase domains ${ }^{12}$ or antisense-mediated downregulation of Flt1 suppresses tumor angiogenesis and VEGF-induced angiogenesis $^{13}$. As Flt1 and PlGF have a restricted angiogenic activity 
$a$
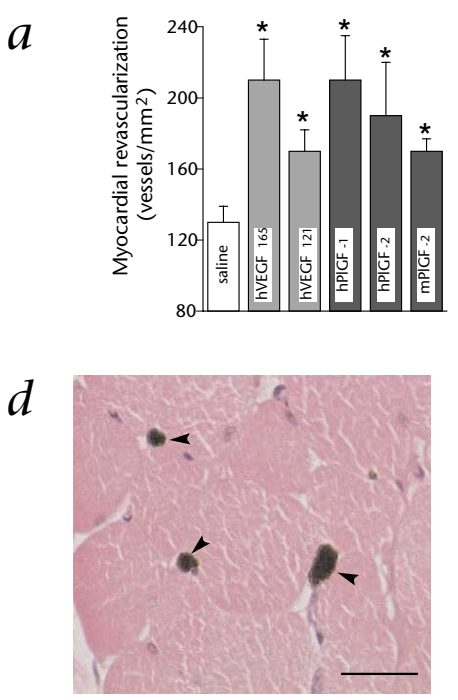

$b$

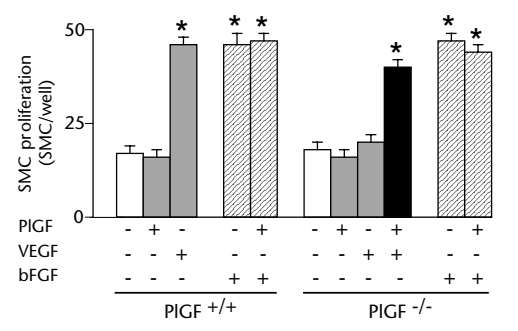

C

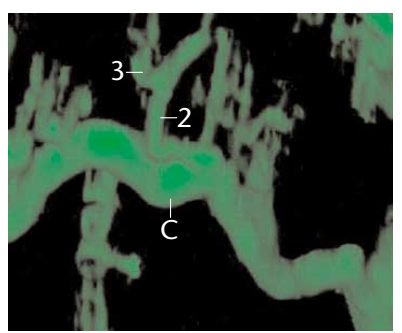

$e$

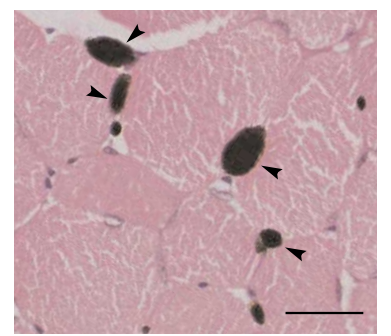

$f$

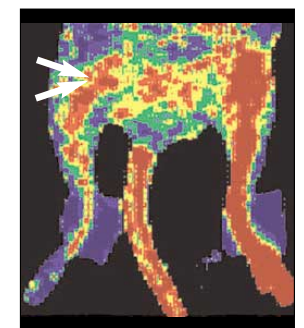

$h$

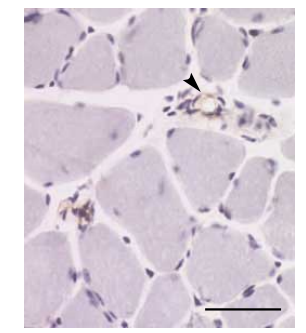

$g$

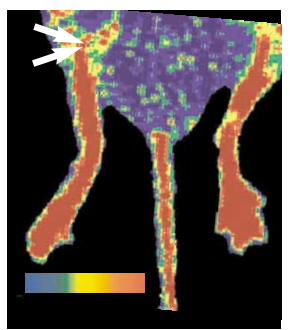

Fig. 1 Therapeutic angiogenesis and arteriogenesis with PIGF in the ischemic myocardium and limb. $\boldsymbol{a}$, Therapeutic myocardial angiogenesis with VEGF or PIGF isoforms (thrombomodulin was used to quantify all vessels). ${ }^{*}, P$ $<0.05 ; n=7-10 . \boldsymbol{b}$, VEGF stimulates proliferation of wild-type $\left(\mathrm{PIGF}^{+/+}\right) \mathrm{SMCs}$ to comparable levels as bFGF (left panel). PIGF is ineffective itself and does not modulate the effect of bFGF. VEGF fails to induce growth of PIGF deficient $\left(\mathrm{PIGF}^{-/}\right)$SMCs whereas PIGF, ineffective itself, amplifies the mitogenic response to VEGF (right panel). This synergism between VEGF and PIGF is specific, as PIGF failed to enhance bFGF-induced SMC proliferation. c, Fluoroangiography, revealing a primary collateral artery (C) with secondary (2) and tertiary (3) collateral side branches. $\boldsymbol{d}$ and $\boldsymbol{e}, \mathrm{H} \& \mathrm{E}$ staining of a section through the adductor muscle, revealing enlarged collateral side branches (arrowheads) after hPIGF-2 treatment (e) as compared with control (d). The lumen of the collaterals is filled with dark bismuth gelatin. $\boldsymbol{f}$ and $\boldsymbol{g}$, Laser doppler imaging, revealing that perfusion through the ligated (arrows) right limb $7 \mathrm{~d}$ after femoral artery ligation is increased by treatment with hPIGF-2 $(g)$ as compared with saline $(f)$. $\boldsymbol{h}$ and $\boldsymbol{i}$, Immunostaining revealing upregulated Flt1 expression in collateral vessels after ligation $(i)$ as compared with control $(h)$. Scale bars, $50 \mu \mathrm{m}$. in pathological conditions, they are potential therapeutic candidates for pro- or anti-angiogenesis. Therefore, we investigated the therapeutic potential of PlGF for stimulation of angiogenesis in ischemic tissues and of Flt1-inhibitors for inhibition of prototypic angiogenic disorders such as cancer, retinal ischemia, arthritis and atherosclerosis.

\section{PIGF stimulates angiogenesis in ischemic myocardium}

The therapeutic potential of PlGF to stimulate angiogenesis was evaluated in a mouse model of ischemic myocardial revascularization $^{11}$. As both VEGF and PlGF exist as multiple isoforms that differ in molecular mass, solubility and receptor binding, the effect of the PlGF isoforms hPlGF-1, hPlGF-2 and mPlGF-2 was compared with that of the VEGF isoforms hVEGF $_{165}$ and $\mathrm{hVEGF}_{121}$. All forms of VEGF and PlGF stimulated the growth of new vessels by $40-60 \%$ (Fig. 1a). We previously documented that PlGF enhanced the VEGF response of PlGF-deficient $\left(\mathrm{PlGF}^{-/}\right)$endothelial cells $(\mathrm{ECs})^{11}$. Therefore, PlGF may induce myocardial revascularization by amplifying the angiogenic activity of VEGF, which is upregulated in the ischemic myocardium $^{11}$. In addition, PlGF could stimulate myocardial angiogenesis by increasing VEGF expression by fibroblasts, which are abundant in the myocardial stroma $\left(\mathrm{pg} / \mathrm{ml}\right.$ per $1 \times 10^{6}$ cells per $24 \mathrm{~h}:<10$ after saline versus $1,500 \pm 450$ after $100 \mathrm{ng} / \mathrm{ml}$ hPlGF-2; $n=6 ; P<0.05$ ). PlGF also stimulated arteriogenesis, or the maturation of vessels via coverage with smooth-muscle cells (SMCs), which leads to stabilization and durability of new ves- sels given that $25-30 \%$ of the new myocardial vessels stained positively for the SMC $\alpha$-actin marker after treatment with all PlGF isoforms. As a similar fraction of myocardial vessels is normally covered by SMCs (25\%), PlGF did not cause 'hemangiomagenesis' but created a new myocardial vasculature with normal characteristics. The functionality of the new vasculature was shown by the improved perfusion of the ischemic myocardium after PlGF treatment $(1,300 \pm 200 \mu \mathrm{l} / \mathrm{min} / \mathrm{g}$ after saline versus $2,100 \pm 190 \mu \mathrm{l} / \mathrm{min} / \mathrm{g}$ after hPlGF-2; $n=7 ; P<0.05)$. Although PlGF and VEGF might affect SMCs indirectly via release of SMC mitogens from activated ECs, they could also stimulate SMCs directly, since both Flt1 and Flk1 were detectable on SMCs by western blotting (data not shown and ref. 14) and by immunostaining in vivo (see below). PlGF determined the responsiveness of SMCs to VEGF, as $\mathrm{PlGF}^{-/}$SMCs only proliferated normally in response to VEGF when PIGF was present (Fig. 1b). PlGF did not affect wild-type SMCs, because their response to exogenous PlGF was obscured by endogenous PlGF production. Thus, PlGF stimulated myocardial revascularization as efficiently as VEGF, but unlike VEGF, PIGF did not cause edema or hypotension (data not shown).

\section{PIGF stimulates arteriogenesis in ischemic limbs}

The potential of PlGF to stimulate the growth of preexisting arterial collaterals and their second- and third-generation side branches ('collateral growth') (Fig. 1c) was evaluated by treating mice with PIGF after ligation of their femoral artery. Delivery of 
$1.5 \mu \mathrm{g}$ hPlGF-2 per day minimally affected the primary collaterals and capillaries in the adductor region, but significantly increased the number and size of the second- and third-generation collateral branches, thereby enlarging the collateral perfusion area (sum of the luminal areas of all secondary and tertiary collaterals) (Table 1 and Fig. $1 d$ and $e$ ). PlGF treatment increased the number of precapillary arterioles, which regulate vascular resistance and tissue perfusion (for example, the collateral sidebranches $>300 \mu \mathrm{m}^{2}$ ) (Table 1). As a result, hind-limb perfusion, determined by laser doppler or microspheres, was increased more than three-fold by PIGF treatment (Table 1 and Fig. $1 f$ and g). Importantly, PlGF treatment improved the spontaneous mobility and the functional muscle reserve of the ischemic hind limb, evaluated using a novel swim endurance exercise test (Table 1). In contrast, a similar dose of hVEGF $_{165}$ was less efficient and consistent in inducing structural and perfusional changes and did not improve the functional swim endurance reserve.

We investigated the arteriogenic mechanisms of PlGF, and its effect on SMCs and macrophages, which are known to produce SMC/EC-mitogens and cytokines during collateral growth ${ }^{15,16}$. Flt1 expression was significantly upregulated in collateral vessels after femoral artery ligation (Fig. $1 h$ and $i$ ). PlGF treatment stimulated SMC growth, as shown by the increased muscular thickness of the collateral branches $(2.35 \pm 0.08 \mu \mathrm{m}$ after saline versus $3.0 \pm 0.2 \mu \mathrm{m}$ after PlGF; $n=9 ; P<0.05)$. PlGF also recruited macrophages around the collateral side branches $\left(\mathrm{Mac}^{+}\right.$ cells/optical field: $3.3 \pm 0.8$ and $3.5 \pm 0.6$ after saline and VEGF, respectively versus $5.8 \pm 0.8$ after PlGF; $n=12 ; P<0.05$ versus saline and VEGF). Moreover, PIGF upregulated the production by macrophages of tumor necrosis factor- $\alpha$ (TNF- $\alpha$ ) and monocyte chemoattractant protein-1 (MCP-1), cytokines implicated in collateral growth ${ }^{17,18}\left(\mathrm{pg} / \mathrm{ml} \mathrm{MCP}-1\right.$ per $4 \times 10^{5}$ macrophages: $81 \pm 7$ after saline versus $101 \pm 8$ after PlGF; pg/ml TNF- $\alpha$ per $4 \times$ $10^{5}$ macrophages: $580 \pm 30$ after saline versus $1,200 \pm 40$ after PlGF; $n=3 ; P<0.05)$. Thus, compared with VEGF, PlGF more efficiently stimulated the functional recovery of the ischemic limb, primarily by enhancing growth of collateral side branches.

\section{PIGF stimulates the formation of mature non-leaky vessels}

The cellular mechanisms of PlGF-induced vessel growth were studied by adenoviral PIGF gene transfer into the skin of ears ${ }^{19}$. Control vectors failed to affect skin vasculature ${ }^{19}$, but PlGF gene transfer caused preexisting vessels to enlarge, resulting in tortuous, thin-walled, pericyte-poor 'mother' vessels (Fig. $2 a-e$ ). The latter vessels remained enlarged and subsequently stabilized into mature durable vessels by acquisition of a pericyte coat and, occasionally, by deposition of a thin rim of perivascular collagen (Fig. 2a-e). Notably, these vessels remained functional and persisted for over one year after PlGF gene transfer, even though transgenes are only transiently expressed for four weeks in this model $^{19}$. Whereas both PIGF and VEGF stimulated the formation of large stabilized vessels, PIGF avoided the harmful complications of VEGF such as edema, fibrin deposition and the growth of unstable vascular tangles and glomeruloid bodies ('hemangioma-genesis') $)^{19,20}$.

\section{Anti-Flt1 inhibits pathological angiogenesis}

To study whether inhibition of Flt 1 would inhibit angiogenesis in ischemic, malignant and inflammatory disorders, monoclonal anti-Flt1 antibody MF1 (anti-Flt1), which blocked binding of VEGF and PlGF to Flt1 (Supplementary Fig. A online) and VEGF- or PlGF-driven growth of ECs (ref. 11), was compared with control IgG and monoclonal anti-Flk1 antibody DC101 (anti-Flk1). Anti-Flt1 efficiently suppressed VEGF-driven neovascularization in the cornea and in matrigel implants (Fig. $2 f-h$ ). It also blocked neovascularization in the ischemic retina $(120 \pm 18$ ECs/10 sections and $44 \pm 7$ vascular tufts/10 sections after control IgG versus $49 \pm 9 \mathrm{ECs} / 10$ sections and $18 \pm 4$ vascular tufts/10 sections after anti-Flt1; $n=19 ; P<0.005$ ) (Fig. $2 i$ and $j$ ) to a comparable degree as genetic deficiency of PlGF (ref. 11) or inhibition of Flk1 (ref. 21). Anti-Flt1 dose-dependently blocked

Table 1 PIGF induces arteriogenesis in ischemic hindlimbs

\begin{tabular}{|c|c|c|c|}
\hline & Saline & PIGF & VEGF \\
\hline \multicolumn{4}{|l|}{ Mean collateral lumen area $\left(\mu \mathrm{m}^{2}\right)$} \\
\hline Collateral arteriole & $1,760 \pm 280$ & $1,710 \pm 210$ & $1,700 \pm 230$ \\
\hline Second-generation collateral side branch & $550 \pm 40$ & $830 \pm 90^{*}$ & $610 \pm 53$ \\
\hline Third-generation collateral side branch & $130 \pm 5$ & $160 \pm 5^{*}$ & $150 \pm 8^{*}$ \\
\hline \multicolumn{4}{|l|}{ Collateral side branches (photoangiography) } \\
\hline Second-generation collateral side branches $(\mathrm{n} / \mathrm{cm})$ & $21 \pm 2$ & $36 \pm 4^{*}$ & $21 \pm 2$ \\
\hline Third generation collateral side branches $(n / \mathrm{cm})$ & $43 \pm 5$ & $65 \pm 9^{*}$ & $40 \pm 7$ \\
\hline \multicolumn{4}{|l|}{ Secondary and tertiary collateral branches } \\
\hline Mice with $>30 \%$ of collaterals $>300 \mu \mathrm{m}^{2}$ & $5 / 14$ & $12 / 14^{*}$ & $7 / 16$ \\
\hline Total perfusion area $\left(\mu \mathrm{m}^{2} / \mathrm{mm}^{2}\right)$ & $2,670 \pm 270$ & $3,900 \pm 360^{*}$ & $2,300 \pm 280$ \\
\hline \multicolumn{4}{|l|}{ Capillaries } \\
\hline Capillary density in adductor muscle $\left(n / \mathrm{mm}^{2}\right)$ & $3,100 \pm 290$ & $2,800 \pm 240$ & $3,600 \pm 440$ \\
\hline \multicolumn{4}{|l|}{ Limb perfusion } \\
\hline \multicolumn{4}{|l|}{ Laser doppler (\% of non-ligated) } \\
\hline Total hindlimb & $61 \pm 7$ & $89 \pm 4^{*}$ & $90 \pm 6^{*}$ \\
\hline Upper hindlimb & $83 \pm 8$ & $101 \pm 2^{*}$ & $102 \pm 4^{*}$ \\
\hline \multicolumn{4}{|l|}{ Microspheres $\left(\mathrm{ml} \times \mathrm{g}^{-1} \times \mathrm{min}^{-1}\right)$} \\
\hline Adductor muscle (collateral region) & $0.06 \pm 0.01$ & $0.22 \pm 0.07^{\star}$ & $0.13 \pm 0.03^{*}$ \\
\hline Gastrocnemius muscle & $0.12 \pm 0.02$ & $0.35 \pm 0.14^{*}$ & $0.21 \pm 0.07$ \\
\hline \multicolumn{4}{|l|}{ Limb motoric function } \\
\hline Spontaneous mobility (score $0-1$ ) & $0.71 \pm 0.13$ & $1^{*}$ & $1^{*}$ \\
\hline Swim test (\% of baseline) & $36 \pm 5$ & $67 \pm 11^{*}$ & $18 \pm 5^{*}$ \\
\hline
\end{tabular}

The data represent the mean \pm s.e.m. of independent measurements in 11-15 mice $7 \mathrm{~d}$ after ligation of the femoral artery (except in 5 mice for microsphere measurements). Values are for daily delivery of $1.5 \mu \mathrm{g}$ growth factor. ${ }^{*}, P<0.05$ versus saline. 
angiogenesis and growth of human epidermoid A431 tumors in nude mice, and was only slightly less active than anti-Flk1 (Fig. $2 k$ ). Compared with the large, vascularized control tumors, anti-Flt1-treated tumors were pale and poorly vascularized, and exhibited extensive necrosis, reduced proliferation and increased tumor-cell apoptosis (data not shown). Flt1 was expressed in tumor-associated vessels, but not in malignant tumor cells (data not shown). By 2 weeks of treatment with anti-Flt1, the microvessel density was reduced by $45 \%$ (vessels per optical field: $6 \pm 1$ in anti-Flt 1 treated tumors versus $11 \pm 2$ in control tumors; $P<0.05$ ) and the vessel size by $30 \%$ (vessels with a luminal diameter $>30 \mu \mathrm{m}$ per optical field: $9.5 \pm 2$ after control versus 3.5 \pm 0.5 after anti-Flt $1 ; P<0.05$ ) (Fig. $2 l$ and $m$ ). Anti-Flt 1 also atten- uated the growth and vascularization of PlGF- or VEGF-transduced rat C6 gliomas implanted in nude mice (Supplementary Fig. B online).

\section{Anti-Flt1 reduces atherosclerotic-plaque growth and vulnerability} Neovascularization of atherosclerotic plaques has been proposed to accelerate lesion growth and to make plaques more vulnerable to rupture, which could trigger fatal thrombotic complications $^{22,23}$. Angiogenesis inhibitors might be useful, but have not been thoroughly studied ${ }^{23}$, and the effect of VEGF receptor inhibitors has not been evaluated. Therefore, initial (avascular) fatty-streak lesions and advanced (vascular) complex plaques were analyzed in atherosclerosis-prone
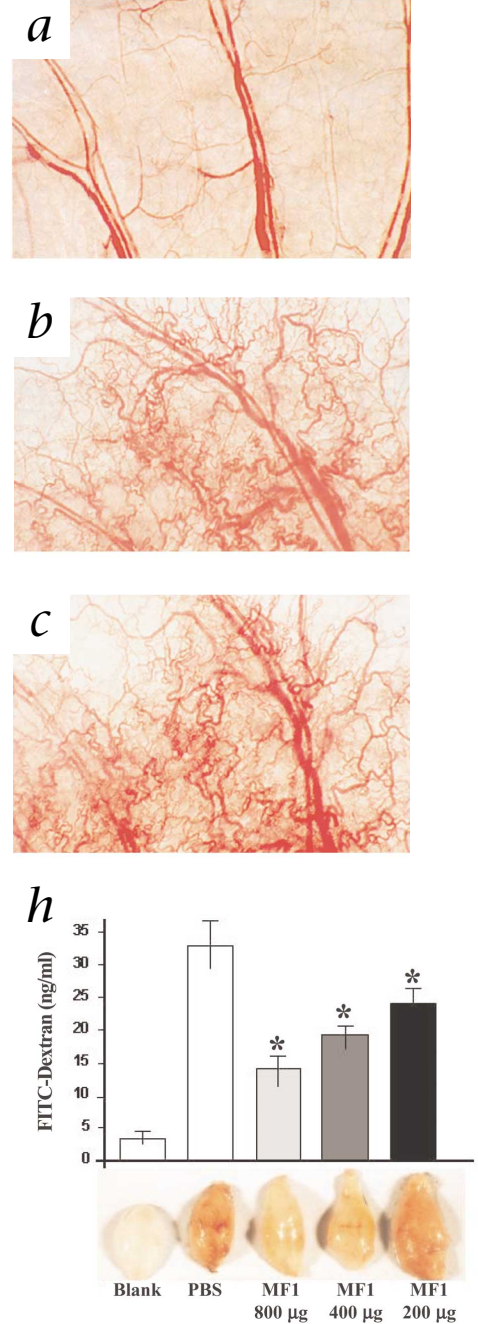

Fig. 2 PIGF stimulates, whereas anti-Flt1 inhibits vessel growth. $\boldsymbol{a}-\boldsymbol{c}$, Vascular response to adenoviral PIGF gene transfer in the ear of a nude mouse: numerous large vessels formed by $14(b)$ and $28 \mathrm{~d}(c)$, and persisted for more than 1 y (data not shown). Note the absence of hemorrhage or edema. $\boldsymbol{d}$ and $\boldsymbol{e}$, Thin-walled, pericyte-poor 'mother' vessels ( $M$ in $d$ ) were stabilized beyond $14 \mathrm{~d}(35 \mathrm{~d}, e)$ by acquisition of pericytes (arrowheads in $e$ ) and small amounts of perivascular collagen. $\boldsymbol{f}$-h, Anti-Flt1 $(g)$ inhibited corneal neovascularization after implantation of a VEGF pellet $(f$ and $g$ ) or dose-dependently inhibited ingrowth of capillaries in matrigel implants, supplemented with VEGF $(h) .{ }^{*}, P<0.05$ versus PBS. $\boldsymbol{i}$ and $\boldsymbol{j}$, H\&E staining of retinal crosssections, revealing fewer ECs (arrowheads) in intravitreal neovessels on the retinal surface after treatment with anti-Flt1 ( () than control $\operatorname{lgG}(I)$. $\boldsymbol{k}$, Anti-Flt1 treatment $(1 \mathrm{mg})$ reduced tumor growth and vascularization as efficiently as anti-Flk1 $(0.8 \mathrm{mg})$. Average tumor volume \pm s.e.m. in groups of 10 nude mice implanted with A431 tumor cells. I and $\boldsymbol{m}$, CD31 immunostaining, revealing reduced vessel density and size in A431 tumors from mice treated with anti-Flt1 $(m)$ as compared with PBS control $(D)$. Vessels are indicated with arrowheads. 'MF-1' in ( $h$ and $k$ ) is anti-Flt1. 'DC101' in $(k)$ is anti-Flk1. Scale bars, $40 \mu \mathrm{m}$ in $d$ and $e$ and $50 \mu \mathrm{m}$ in $i$ and $j$.

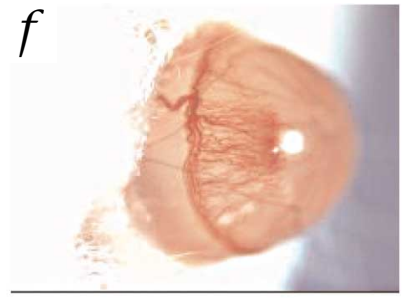

Vascular index: $0.45 \pm 0.05$

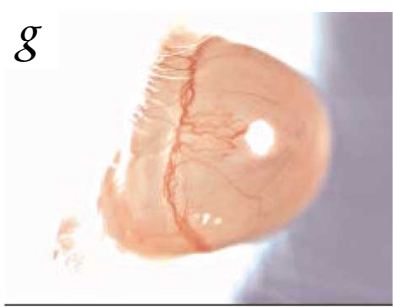

Vascular index: $0.21 \pm 0.04$ 
Table 2 Effects of anti-Flt1 on atherosclerosis and mobilization of hematopoietic progenitors

\begin{tabular}{|c|c|c|}
\hline & Control lgG & Anti-Flt1 \\
\hline \multicolumn{3}{|l|}{ Advanced atherosclerosis } \\
\hline \multicolumn{3}{|l|}{ Inflammation and matrix deposition } \\
\hline Total $\mathrm{Mac}^{+}$plaque area $\left(\mu \mathrm{m}^{2} \times 10^{3}\right)$ & $180 \pm 18$ & $110 \pm 23^{*}$ \\
\hline CD45 ${ }^{+}$adventitial area (\%) & $11 \pm 0.5$ & $8 \pm 0.6^{*}$ \\
\hline Total Sirius red ${ }^{+}$collagen area $\left(\mu \mathrm{m}^{2} \times 10^{3}\right)$ & $350 \pm 30$ & $330 \pm 35$ \\
\hline \multicolumn{3}{|l|}{ Angiogenesis } \\
\hline Number of plaque vessels per mouse & $1.5 \pm 0.5$ & $1.4 \pm 0.4$ \\
\hline Proportion of mice with at least 1 plaque with vessels (\%) & $60(15)$ & $53(15)$ \\
\hline Proportion of plaques with at least 1 vessel (\%) & $21(52)$ & $23(52)$ \\
\hline Adventitial vessel density $\left(n / \mathrm{mm}^{2}\right)$ & $370 \pm 2$ & $400 \pm 23$ \\
\hline \multicolumn{3}{|l|}{ Circulating monocytes and granulocytes } \\
\hline \multicolumn{3}{|l|}{ CD11 $b^{+} / G r-1^{\text {Lo }}$ monocytes } \\
\hline Control mice & $3.3 \pm 0.3$ & $3.9 \pm 1.1$ \\
\hline Atherosclerotic mice & $8 \pm 0.7$ & $5.5 \pm 0.5^{*}$ \\
\hline Arthritic mice & $14 \pm 2$ & $8.5 \pm 1.1^{*}$ \\
\hline \multicolumn{3}{|l|}{ CD $11 b^{+} / G r-1^{\mathrm{Hi}}$ granulocytes } \\
\hline Control mice & $20 \pm 1.5$ & $20 \pm 3.2$ \\
\hline Atherosclerotic mice & $50 \pm 5$ & $35 \pm 4^{*}$ \\
\hline Arthritic mice & $44 \pm 4$ & $32 \pm 2^{*}$ \\
\hline \multicolumn{3}{|l|}{ Circulating hematopoietic progenitors (after G-CSF) } \\
\hline Erythroid burst forming units (b.f.u.-E) & $2.2 \pm 1.0$ & $0.1 \pm 0.1 *$ \\
\hline Total myeloid c.f.u. & $24 \pm 5$ & $6 \pm 2^{*}$ \\
\hline Granulocyte c.f.u. & $7.3 \pm 2.5$ & $1.6 \pm 0.5^{*}$ \\
\hline Monocyte c.f.u. & $5.4 \pm 1.7$ & $0.9 \pm 0.4^{*}$ \\
\hline Granulocyte/monocyte c.f.u. & $11.6 \pm 1.4$ & $2.6 \pm 0.9^{*}$ \\
\hline Mixed c.f.u. & $1.6 \pm 0.7$ & $0.1 \pm 0.1^{*}$ \\
\hline \multicolumn{3}{|c|}{$\begin{array}{l}\text { The data represent mean } \pm \text { s.e.m. of mice ( } 9-15 \text { for atherosclerosis; } 10 \text { for c.f.u. assays; } 6-10 \text { for circulating leuko- } \\
\text { cytes). }{ }^{*}, P<0.05 \text { by unpaired Student's } t \text {-test versus control IgG. Plaque analyses were performed on } 6-10 \text { cross- } \\
\text { sections per mouse at the aortic root. The number of plaques or mice is indicated in parentheses. Baseline levels of } \\
\text { hematopoietic progenitors in the peripheral blood without G-CSF were undetectable. Circulating granulocytes and } \\
\text { monocytes are expressed in percentages. Number of c.f.u. is expressed per } 100 \mu \text { l blood. }\end{array}$} \\
\hline
\end{tabular}

from where vessels sprout into plaques ${ }^{22,23}$ (Table 2). This effect was specific, as antiFlt1 did not affect collagen deposition (Table 2). Vessels in plaques and in the overlying adventitia were identified on semi-thin sections and on histological sections by immunostaining for the endothelial markers CD31, von Willebrand factor and Flk-1 (Fig. $3 i$ and $j$ ). In contrast to the abundant angiogenesis in the adventitia at all disease stages, neovascularization within atherosclerotic lesions was only detectable at advanced stages. To reliably quantify the minimal intra-plaque neovascularization, both the vascular density and incidence of mice and plaques with vessels were counted. Based on the antiangiogenic effect of the anti-Flt1 in tumors and ischemic retina (see above), we had anticipated that the reduced plaque growth might, at least in part, be attributable to inhibition of plaque neovascularization. Surprisingly, however, anti-Flt1 failed to affect angiogenesis in the plaque and in the adventitia (Table 2). Thus, antiFlt1 suppressed plaque growth and vulnerability via inhibition of inflammatory cell infiltration (and myeloid progenitor mobilization; see below), independently of angiogenesis, whereas anti-angiogenic anti-Flk1, which normally blocks angiogenesis, was ineffective.
apolipoprotein-E-deficient $\left(\mathrm{ApoE}^{-/}\right)$mice. Expression of VEGF, and in particular of PlGF, was upregulated in early and advanced lesions (pg/ml: $18 \pm 7,50 \pm 9$ and $40 \pm 5$ for VEGF and $70 \pm 28,210 \pm 38$ and $380 \pm 60$ for PlGF in non-atherosclerotic aorta, fatty streaks and advanced lesions, respectively; $n=6$ to $10 ; P<0.05$ versus normal aorta). PIGF and VEGF were detectable in cells in the adventitia, in the plaque shoulders and cap (Fig. $3 a$ and $b$ ), whereas Flt1 was expressed by SMCs of the vessel wall and by ECs in adventitial vessels and luminal ECs (Fig. 3c). Flk1 was only detectable in capillaries in the adventitia and in intra-plaque vessels (Fig. 3j). To study the role of Flt1 and Flk1 in early, intermediate or advanced plaque lesions, $\mathrm{ApoE}^{-/-}$mice were treated with control IgG, anti-Flt1 or antiFlk1 for 5 weeks starting at 5, 10 or 20 weeks of age. Treatment with anti-Flt1 reduced the size of early and intermediate lesions at the aortic root by $50 \%$ (Fig. $3 d$ ) and the growth of advanced atherosclerotic lesions by $\sim 25 \%$ (plaque size: $770,000 \pm$ $40,000 \mu \mathrm{m}^{2}$ after IgG versus $600,000 \pm 50,000 \mu \mathrm{m}^{2}$ after antiFlt $1 ; n=10, P=0.03)$. Notably, the anti-Flk1 did not affect atherosclerotic plaque development at all stages (early and intermediate plaques (Fig. $3 d$ ); advanced plaques: 700,000 \pm $60,000 \mu \mathrm{m}^{2} ; n=10, P=$ n.s. versus IgG).

To examine the cellular mechanisms of the atheroprotective effect of anti-Flt1, lesions were stained with specific markers. Anti-Flt1 reduced, by $40 \%$, macrophage infiltration into early lesions (total $\mathrm{Mac}^{+}$plaque area: 37,000 $\pm 2,000 \mu \mathrm{m}^{2}$ after IgG versus $15,300 \pm 800 \mu \mathrm{m}^{2}$ after anti-Flt1; $n=10 ; P=0.01$ ) as well as in advanced lesions (Table 2 ; Fig. $3 e$ and $f$ ). Anti-Flt1 also suppressed macrophage-driven inflammation in the adventitia,

\section{Anti-Flt1 suppresses autoimmune arthritic joint destruction}

Angiogenesis may contribute to the proliferation of synoviocytes, infiltration of inflammatory cells, cartilage destruction and pannus formation-all hallmarks of rheumatoid arthritis $^{24,25}$. VEGF is upregulated in arthritic joints and neutralization of VEGF reduces joint destruction ${ }^{26,27}$, but inhibitors of VEGF receptors have not been evaluated. To study the therapeutic potential of anti-Flt1 and anti-Flk1, polyarticular arthritis was induced in mice, using an autoimmune model of collagen type II-induced arthritis (a model for rheumatoid arthritis in humans $^{28}$ ). Immunostaining of affected joints revealed that VEGF was present in inflammatory cells, chondrocytes and cells at the pannus-bone interface and on ECs in synovial neovessels, whereas Flk1 was only present in synovial neovessels (Supplementary Fig. C online). Flt1 and PlGF were expressed by inflammatory and, probably, also by ECs in the inflamed synovium. Treatment with anti-Flt1 reduced the incidence of joint disease by $60 \%$, whereas all IgG-treated mice developed signs of arthritis in the paws and ankles (Fig. $4 a$ ). Notably, anti-Flt1 treatment suppressed the development of clinical symptoms (paw swelling, erythema and ankylosis) by $85 \%$ (clinical score: $1.2 \pm$ 0.6 after anti-Flt1 versus $7.5 \pm 1.2$ after IgG; $n=24, P<0.0001$ ) (Fig. $4 b$ ). The effect observed with treatment with anti-Flt1 was specific, since anti-Flk1 was ineffective (Fig. $4 a$ and $b$ ). The antiarthritic effect of anti-Flt1 was not attributable to changes in humoral or cellular immunity.

The histological disease severity score for each joint-a measure of the degree of synovial hyperplasia, inflammation and pannus formation-was lower after anti-Flt1 treatment (Fig. 4c). 
Anti-Flt1 also suppressed the degradation of cartilage proteoglycans (safranin $\mathrm{O}^{+}$area per $\mathrm{mm}$ talus/calcaneus interface: $91 \pm$ $4 \mu \mathrm{m}^{2}$ after anti-Flt1 versus $64 \pm 7 \mu \mathrm{m}^{2}$ after IgG; $n=9 ; P<0.05$ ) (Fig. $4 d$ and $e$ ). Synovial infiltration by inflammatory cells was reduced by anti-Flt1 treatment $\left(\mathrm{CD} 45^{+}\right.$or $\mathrm{Mac}^{+}$leukocyte area: $8 \pm 2 \%$ and $8 \pm 2 \%$ after anti-Flt1 versus $20 \pm 3 \%$ and $16 \pm 2 \%$ after IgG, respectively; $n=9 ; P<0.05$ ) (Fig. $4 f$ and $g$ ). In addition, anti-Flt1 inhibited synovial angiogenesis $\left(\mathrm{CD} 31^{+}\right.$vessels $/ \mathrm{mm}^{2}$ : $340 \pm 60$ after anti-Flt 1 versus $530 \pm 25$ after IgG; $n=9 ; P<0.05$ ) (Fig. $4 h$ and $i$ ). The anti-Flt 1 might be anti-inflammatory, in part because it suppressed activation of leukocytes. Indeed, anti-Flt1 suppressed the production of TNF- $\alpha$ and MCP-1, cytokines implicated in arthritis ${ }^{24}$, by PlGF-activated macrophages $(\mathrm{pg} / \mathrm{ml}$ TNF- $\alpha$ per $4 \times 10^{5}$ macrophages: $1,200 \pm 40$ after IgG versus 1,000 \pm 90 after anti-Flt $1 ; \mathrm{pg} / \mathrm{ml}$ MCP- 1 per $4 \times 10^{5}$ macrophages: $100 \pm$ 8 after IgG versus $70 \pm 6$ after anti-Flt1; $n=3 ; P<0.05$ ). Moreover, anti-Flt1 inhibited adhesion of neutrophils (adherent cells per $\mathrm{mm}^{2}: 31 \pm 1$ after anti-Flt1 versus $63 \pm 3$ after IgG; $n=4$; $P<0.05)$. Thus, inhibition of Flt1, but not of Flk1, protected against arthritic joint destruction by suppressing synovial inflammation and neovascularization.

\section{Anti-Flt1 blocks mobilization of myeloid progenitors}

The failure of anti-Flk1, but not of anti-Flt1, to block arthritis $a$

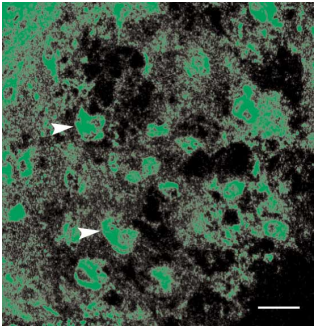

$e$

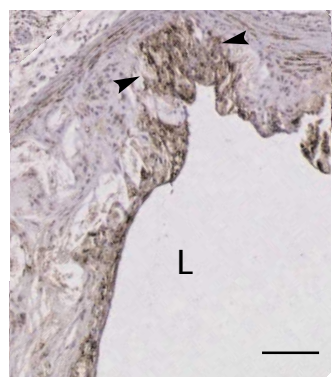

$b$

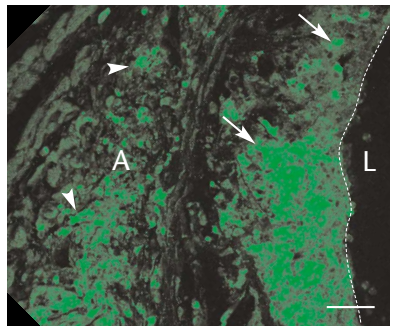

$f$

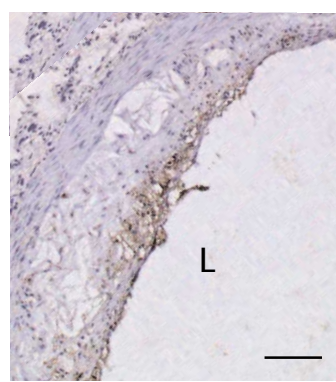

Fig. 3 Anti-Flt1 mAb inhibits inflammation but not angiogenesis in atherosclerotic plaques. $\boldsymbol{a}-\boldsymbol{c}$, Immunostaining of PIGF $(a)$ and VEGF $(b)$ in an advanced plaque, and of Flt 1 in an early plaque (c). PIGF and VEGF are expressed in luminal ECs, adventitial inflammatory cells (arrowheads in $b$ ) and cells in the plaque shoulders (arrowheads in (a)) and cap (arrows in $b$ ); Flt 1 is expressed in adventitial capillaries (arrowhead in $c$ ), SMCs and luminal ECs (arrow in c). IEL: internal elastic lamina; EEL: external elastic lamina. d, Anti-Flt1 (black), but not anti-Flk1 (gray) suppressed growth of early and intermediate atherosclerotic plaques by $50 \%$. ${ }^{*}, P<0.05$ versus control $\lg G$ (white). $\boldsymbol{e}$ and $\boldsymbol{f}$, Mac3 staining of advanced plaques (aortic root), revealing that anti-Flt1 reduced macrophage accumulation $(f)$ as compared with control IgG (e). Note the intense staining at the plaque shoulder in the $\lg G$ plaque (arrowheads) (e). $\boldsymbol{g}$ and $\boldsymbol{h}$, Lateral view of an intercostal artery, containing an early atherosclerotic plaque at its ostium, where the intercostal artery branches off from the aorta. The green cells inside the plaque are GFP ${ }^{+}$ bone marrow-derived cells, which infiltrated into the plaques after transplantation. Infiltration of $\mathrm{GFP}^{+}$cells (of which $66 \pm 4 \%$ were macrophages) in plaques was inhibited by anti-Flt1 $(h)$ as compared and atherosclerosis and the angiogenesis-independent atheroprotective effect of the anti-Flt1 indicated that suppression of inflammation, not angiogenesis, was primarily responsible for the observed effects. Therefore, the effect of Flt1 on myeloid cells was further examined. Transplantation of bone marrow, transduced with a retroviral green fluorescent protein (GFP)expressing vector, revealed that anti-Flt1 blocked the accumulation of GFP-labeled bone marrow-derived cells in atherosclerotic lesions (GFP ${ }^{+}$cells per lesion: $12 \pm 2$ after anti-Flt1 versus $31 \pm 7$ after IgG; $n=5 ; P<0.05$ ) (Fig. $3 g$ and $h$ ). By Mac3 staining, $66 \pm$ $4 \%$ of these cells were identified as macrophages. Similar results were obtained in the arthritis model (data not shown). Reduced leukocyte accumulation could result from an effect of anti-Flt1 on the infiltration of circulating myeloid cells in inflamed lesions and/or from an effect on the differentiation or mobilization of these cells or their progenitors from the bone marrow into the peripheral blood. In support of the latter mechanism, anti-Flt1 partially abrogated the disease-associated increase in circulating monocytes and granulocytes (Table 2). In addition, mobilization of progenitors was suppressed by anti-Flt1. As plasma levels of granulocyte colony-stimulating factor (G-CSF) and of granulocyte-macrophage colony-stimulating factor (GMCSF) increase during inflammation and induce the expansion of myeloid progenitors in the bone marrow and their mobilization
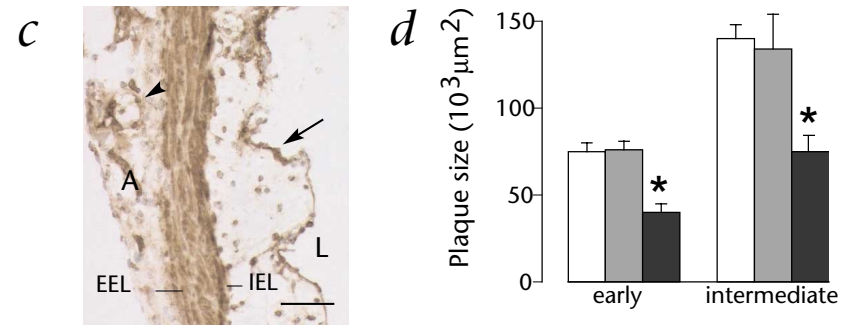

$g$

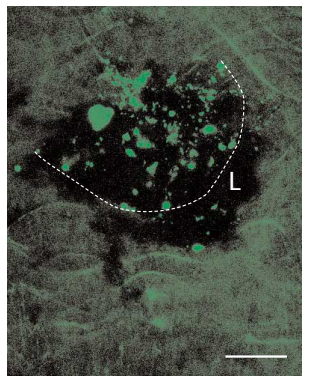

$h$

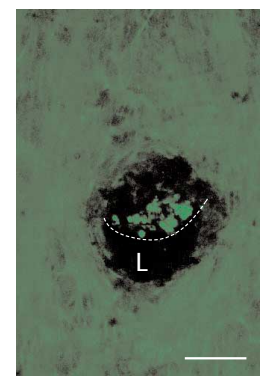

\section{$i$}
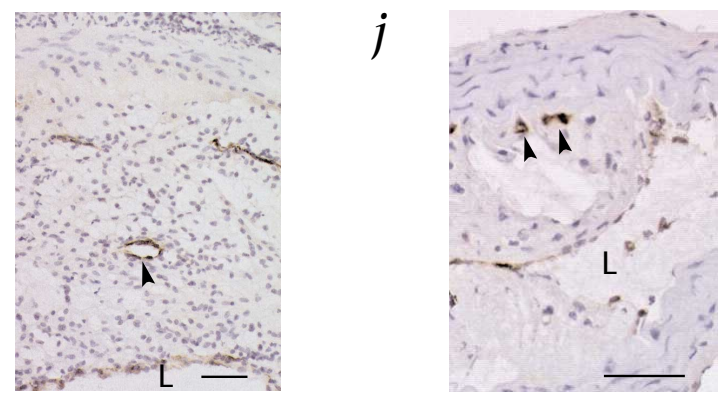

with control IgG $(g)$. $\boldsymbol{i}$ and $\boldsymbol{j}$, Advanced atherosclerotic lesions, stained for von Willebrand factor (i) or Flk1 $(j)$, revealing the presence of plaque vessels (arrowheads). $\mathrm{L}$ in $b, c$ and $e-j$ indicates the vessel lumen. Dashed white lines in $b, g$ and $h$ indicate the luminal plaque endothelium; dashed red lines in $a, b, g$ and $h$ indicate the internal elastic lamina lining the plaque base. Scale bars, $100 \mu \mathrm{m}$ in $(a), 10 \mu \mathrm{m}(g$ and $h)$, $50 \mu \mathrm{m}(c, b, e$ and $f$ ) and $25 \mu \mathrm{m}$ ( $i$ and $j)$. 

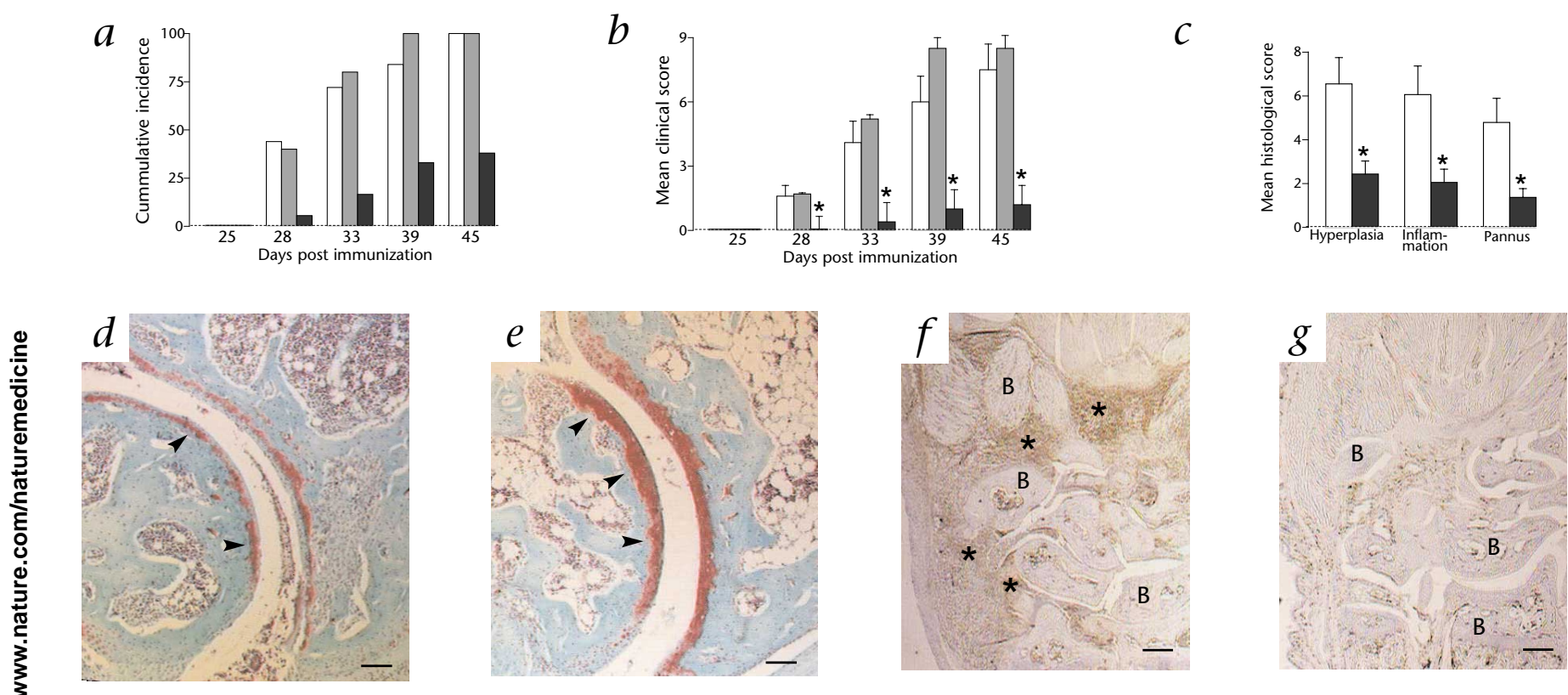

Fig. 4 Anti-Flt1, but not anti-Flk1, inhibits arthritic joint destruction. $a$, Anti-Flt1 ( $\mathbf{\square})$ reduced the number of mice with clinical signs of arthritis by $>60 \%$ as compared with mice receiving control IgG $(\square)$ or anti-Flk1 ( $\square$ ). $\boldsymbol{b}$, Mean clinical arthritis score in all paws and ankles $(n=20-23)$, revealing significantly reduced disease severity after anti-Flt1 (ם) but not after antiFlk1 ( $\square)$. ${ }^{*}, P<0.05$ versus IgG $(\square)$. c, Histological score of the degree of synovial hyperplasia, inflammation and pannus formation in all paws and ankles, revealing a 3-fold reduction after anti-Flt1 treatment ( $\square)$ as compared with control $\lg G(\square)$. Mean \pm s.e.m. of 10 mice. ${ }^{*}, P<0.05$ versus control IgG. $\boldsymbol{d}$ and $\boldsymbol{e}$, Cross-sections of ankle joints at the talus-tibia interface, revealing more extensive destruction of safranin O-stained (red) proteoglycans in control mice (d) than in anti-Flt1-treated mice (e). Arrowheads indicate cartilage. $\boldsymbol{f}$ and $\boldsymbol{g}$, CD45 staining, revealing more abundant accumulation of $\mathrm{CD} 45^{+}$inflammatory cells (asterisks) in the joints of the fore paw in mice receiving control IgG $(f)$ than in anti-Flt1-treated mice $(g) . \boldsymbol{h}$ and $\boldsymbol{i}$, Staining for CD31, revealing reduced synovial neovascu-
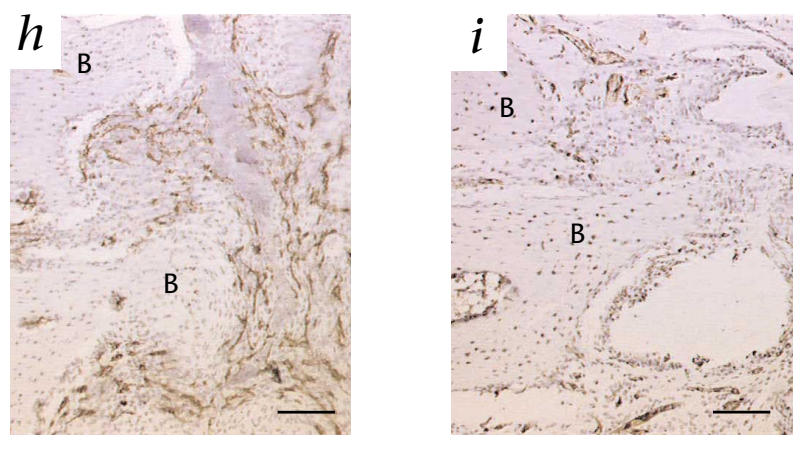

larization after anti-Flt1 treatment $(I)$ as compared with control $\lg G(h)$. 'B' in $(f-l)$ indicates bone. Scale bars, $100 \mu \mathrm{m}$.

from the bone marrow into the blood, we investigated the effect of anti-Flt1 treatment on these G-CSF-induced processes. AntiFlt1 did not affect the G-CSF-induced expansion of myeloid progenitors in the bone marrow (data not shown), but reduced their mobilization into the peripheral blood by $75 \%$ (Table 2 ).

\section{Discussion}

PlGF and its receptor Flt1 have not received much attention as therapeutic targets ${ }^{7,8}$. Here, we report that PlGF stimulates the formation of mature and durable vessels in the ischemic heart, and enlarges collateral arterioles in the ischemic limb with marked perfusional and functional improvement. Conversely, anti-Flt1 blocked excess neovascularization in the ischemic retina and in tumors and protected against arthritis and atherosclerosis, disorders driven by angiogenesis ${ }^{4,29}$. Anti-Flk1 was ineffective in blocking these inflammatory disorders, indicating that targeting Flk1-driven angiogenesis is insufficient to halt disease progression and that the role of VEGF, PIGF and their receptors in these disorders is etiopathogenetically more importantly related to inflammation than to angiogenesis. In support of the latter hypothesis, anti-Flt1 suppressed the mobilization of myeloid progenitors into the peripheral blood, and their differentiation/activation and infiltration into inflamed tissues.
Flt1 and its ligands may stimulate EC growth via several complementary and mutually non-exclusive mechanisms. First, by displacing VEGF from Flt1, PlGF would make more VEGF available to activate Flk1 (ref. 10). Second, PlGF could upregulate expression of VEGF by peri-endothelial fibroblasts, SMCs or inflammatory cells in wound or tumor stroma ${ }^{30}$ (and this study). Third, Flt1 could transmit its own intracellular angiogenic signals ${ }^{8}$. Fourth, PlGF might activate a receptor cross-talk between Flt1 and Flk1, leading to enhanced Flk1-driven angiogenesis (data not shown). The regulation of EC functions by Flt1 may also explain why inhibition of Flt1 efficiently blocked VEGFdriven angiogenesis in the cornea, ischemic retina, arthritic joints and tumors. The observation that anti-Flt1 suppressed pathological angiogenesis comparably to anti-Flk1 indicates that Flt1 is a more important therapeutic target for inhibition of angiogenesis than previously presumed. Others reported that antiFlt1 did not affect tumor growth ${ }^{31}$, but insufficient amounts of antibody may have been used, given that a more complete inhibition of Flt 1 reduced tumor angiogenesis here. In addition, our data extend previous findings that antisense-mediated downregulation of Flt1 suppressed VEGF-driven tumor angiogenesis ${ }^{13}$. Growth of Lewis lung carcinomas (LCC), endogenously expressing VEGF or overexpressing PIGF, was also impaired in mice ex- 
pressing Flt1 without tyrosine kinase domain (Flt1/TK- ${ }^{12}$. When VEGF was overexpressed in LLC, tumor growth was restored, presumably because VEGF levels were sufficiently elevated to drive angiogenesis exclusively via Flk1 (ref. 12). Thus, the relative importance of Flk1- versus Flt1-driven angiogenesis may depend on the relative expression of VEGF, PlGF, Flk1 and Flt1. Surprisingly, inhibition of neither Flt1 nor Flk1 blocked angiogenesis in atherosclerotic lesions. VEGF and PlGF were expressed in plaques, but it remains to be determined whether their proangiogenic activity was overshadowed by other stimulators (bFGF, intergrins) or counterbalanced by angiogenesis inhibitors (thrombospondin-1, TIMPs) in plaques ${ }^{23,32}$.

PlGF and Flt1 also stimulate 'arteriogenesis' by affecting smooth muscle cells (SMCs), which are critical for the establishment of mature, durable and functional neovessels ${ }^{15,16}$. Indeed, vessels covered with SMCs fail to regress and secure persistent tissue perfusion when the angiogenic stimulus fades away ${ }^{33}$. PlGF treatment may achieve this therapeutic goal as it combines two desirable properties; for example, it stimulates SMCs coincidently with ECs and, thereby, may induce vessel growth in a more balanced manner than VEGF or PDGF-BB, which preferentially stimulate ECs or SMCs, respectively ${ }^{15}$. In the ischemic myocardium, PlGF could recruit SMCs by itself ${ }^{14}$ or, as illustrated by the present in vitro findings, in synergy with VEGF. The latter amplification of VEGF by PlGF might explain why PlGF only stimulated revascularization in ischemic but not in healthy tissues.

However, improved perfusion of ischemic tissues does not only rely on the growth of small capillaries ('angiogenesis') and the maturation of these naked endothelial channels with a SMCcoat ('arteriogenesis'), but also on the growth of large arterial collaterals ('collateral growth'), since the latter conduct larger amounts of blood flow ${ }^{15,16}$. Collateral growth in the ischemic limb is dependent on the recruitment of inflammatory cells, which produce EC/SMC mitogens ${ }^{15,16}$. To date, only a few arteriogenic molecules have been identified to affect collateral growth. Compared to bFGF (ref. 34) or MCP-1 (refs. 15,17,18), which preferentially affect either EC/SMCs or inflammatory cells, respectively, PIGF affected the three principal cell types responsible for collateral growth ${ }^{15,16}$ : ECs (ref. 11), SMCs and macrophages, which all expressed increased levels of Flt 1 during collateral growth. Notably, PIGF recruited and activated monocytes to produce increased amounts of MCP- 1 and TNF- $\alpha$, cytokines implicated in collateral growth ${ }^{17,18}$. It remains to be determined whether PIGF also enhanced recruitment of myeloid progenitors but, because anti-Flt1 suppressed this process (see below) and transplantation of wild-type bone marrow rescued the impaired collateral growth in PlGF-deficient mice ${ }^{11}$, such a mechanism appears plausible. Hematopoietic stem cells indeed appear to express Flt1 and to be responsive to PlGF (ref. 35).

Flt1, unlike Flk1, is also expressed by inflammatory cells (ref. 36 and this study). The activity of PlGF and VEGF to attract Flt ${ }^{+}$ leukocytes may explain why anti-Flt1 suppressed inflammatory disorders, whereas anti-Flk1 antibodies were ineffective. PlGF and VEGF, which are upregulated within these inflamed tissues (refs. 26, 27, 30 and this study), may provide recruitment signals for differentiated inflammatory cells to home to and infiltrate sites of ongoing inflammation. However, they may also induce mobilization of myeloid progenitors from the bone marrow to the blood. Our findings that anti-Flt1 attenuated the disease-induced upregulation of circulating leukocytes and the G-CSF induced mobilization of myeloid progenitors support such a mechanism and extend previous findings that anti-Flt1 blocked the recruitment of perivascular Flt $1^{+}$myeloid cells in tumors ${ }^{31}$. Flt1 also seems to be involved in cellular activation, as anti-Flt1 impaired the production of MCP-1 and TNF- $\alpha$ by macrophages. A Flt1-mediated increase in release of proteinase ${ }^{37}$ might further explain why anti-Flt1 suppressed cartilage destruction in arthritic joints. Furthermore, because PlGF upregulates VEGF production by monocytes ${ }^{30}$, inhibition of Flt 1 would reduce VEGF-driven angiogenesis and, secondarily, inflammation. Taken together, a role of Flt1 in mobilization of myeloid progenitors and in migration, activation and, possibly, differentiation of myeloid cells may explain part of the therapeutic effect of anti-Flt1 in inflammatory disorders.

Our findings may have several medical implications. First, inhibition of VEGF-driven angiogenesis has been considered to be an attractive therapy for inflammatory disorders ${ }^{4,23,29,38}$. However, inhibition of the prototype angiogenic Flk1 receptor, which efficiently inhibits tumor angiogenesis, did not block arthritis or atherosclerosis, whereas Flt1-inhibitors did. This implies that blocking Flk1-driven angiogenesis alone, in the absence of anti-inflammatory agents, may not suffice. Second, anti-Flt1 did not only reduce the size but, more importantly, also stabilized atherosclerotic lesions, which might prevent plaque rupture and fatal thrombotic events ${ }^{39}$. This degree of protection by anti-Flt1 is comparable with-or even better than-that achieved by treatment with statins ${ }^{39}$ or other anti-inflammatory compounds ${ }^{40}$. Because anti-Flt1 was only administered for five weeks, an increased efficiency might be possible with chronic administration. Third, inhibition of Flt 1 may provide a novel treatment for the inhibition of tumor angiogenesis and ischemic retinal neovascularization, and of inflammatory diseases. Lastly, PIGF may be an attractive candidate for improved therapeutic angiogenesis and arteriogenesis, as it efficiently stimulated vessel growth in ischemic tissues without affecting quiescent vessels. Importantly, the PlGF-induced mature vessels persisted for prolonged periods ( $>1$ year), even long after the arteriogenic stimulus had disappeared ${ }^{19}$, indicating that it may suffice to stimulate new vessels with a short-term delivery of PIGF. Moreover, PlGF is less likely to cause the side effects in situations where chronic, sustained delivery may be required ${ }^{33,41}$, as it did not cause undesirable side effects of hyperpermeability, edema and hemangioma-genesis associated with VEGF. Clearly, as for all pro- or anti-angiogenic compounds, caution is warranted to avoid aggravation of ischemic tissue disease by uncontrolled use of anti-Flt1, or of atherosclerosis or cancer by systemic delivery of PIGF. Although future studies will need to address in more detail the safety versus efficacy of Flt1-ligands and its inhibitors in other preclinical models, anti-Flt1 did not appear to impair myocardial angiogenesis, wound healing or ovulation (data not shown). In conclusion, these findings provide a rationale for evaluating Flt1 and its ligands as therapeutic targets for promoting revascularization of ischemic tissues and for blocking uncontrolled angiogenesis and inflammation in cancer, arthritis, atherosclerosis and retinal ischemia.

\section{Methods}

Antibodies, gene expression and recombinant proteins. Quantitative real-time RT-PCR and protein extraction were performed as described ${ }^{11}$. Murine VEGF, PIGF, MCP-1 and TNF- $\alpha$ protein levels were quantified by ELISA (R\&D Systems, Abingdon, UK). Rat anti-mouse Flt1 (clone \#MF-1) and rat anti-mouse Flk1 (clone \#DC101) were developed at ImClone Systems Inc. ${ }^{42}$. rhVEGF 165, rhVEGF $_{121}$, rhPIGF-1 and rmPIGF-2 were from $R \& D$ Systems and rh-PIGF-2 from Reliatech (Braunschweig, Germany), 
rhG-CSF from Amgen (Breda, the Netherlands) and control rat IgG from Sigma.

Mouse models of angiogenesis, atherosclerosis or arthritis. Matrigel and mouse corneal micropocket assays with $\mathrm{VEGF}_{165}$ with addition of $1 \mu \mathrm{g}$ control IgG or anti-Flt1 were performed as previously described ${ }^{11} .2 \times 10^{6} \mathrm{~A} 431$ or PIGF- or VEGF-overexpressing rat C6 glioma tumor cells were subcutaneously injected in nude mice. Groups of 10 mice with tumors of comparable size (150-200 $\left.\mathrm{mm}^{3}\right)$ received intraperitoneal injections of anti-Flt1 (200 or $1,000 \mu \mathrm{g})$, anti-Flk $1(800 \mu \mathrm{g})$ or control $\lg \mathrm{G}(1,000 \mu \mathrm{g})$ every $3 \mathrm{~d}$. Tumors were measured with calipers and tumor volumes calculated using the formula $\left[\Pi / 6\left(w_{1} \times w_{2} \times w_{2}\right)\right]$, where ' $w_{1}{ }^{\prime}$ and ' $w_{2}$ ' represent the largest and smallest tumor diameter, respectively. Tumor vessel density and size were determined on CD31-stained sections. Ischemic retinal neovascularization with control IgG or anti-Flt1 ( $400 \mu \mathrm{g}$ per pup; administered from P12 onwards) was analyzed as described ${ }^{11}$. ApoE ${ }^{-1-}$ mice with a mixed genetic background of $75 \% \mathrm{C} 57 \mathrm{BI} / 6$ and $25 \% 129 \mathrm{SvJ}$ were fed a high fat/cholesterol diet at $5 \mathrm{wk}$ of age and intraperitoneally injected 3 times per wk with $500 \mu \mathrm{g}$ anti-Flt1 or $800 \mu \mathrm{g}$ anti-Flk1 for 5 wk starting at wk 5,10 or 20 . Measurement of plasma cholesterol levels, tissue processing, immunostaining and morphometric analysis of lesions at the aortic root and in the dorsal aorta was performed as described ${ }^{43}$. Collagen-induced arthritis (CIA) was performed using DBA/1JOla mice as described ${ }^{44}$. Briefly, mice were immunized on d 0 with $100 \mu \mathrm{g}$ chicken collagen type II (CII) in $100 \mu \mathrm{l}$ complete Freund's adjuvant, containing $150 \mu \mathrm{g}$ heat-killed mycobacterium butyricum and received a booster injection with $50 \mu \mathrm{g} \mathrm{Cll}$ in incomplete Freund's adjuvant on $d 21$. Disease severity was scored for each paw from d 10 as described $^{45}$. Treatment with anti-Flt1, anti-Flk1 or control IgG was initiated at $d$ 10 by intraperitoneal injection of $750 \mu \mathrm{g} /$ mouse at 3-d intervals. At $d 45$, mice were killed for histological analysis using a standard scoring protocol ${ }^{45}$. Infiltration by $\mathrm{CD}^{4} 5^{+}$leukocytes or $\mathrm{Mac}^{+}$macrophages and formation of $\mathrm{CD} 31^{+}$vessels in the synovium were determined in the most severely affected paw by measuring the immunoreactive-area in at least 8 randomly chosen fields per paw. Cartilage degradation was examined on safranin Ostained sections of the most severely affected ankles for each mouse at two different locations (tibia-talus and talus-calcaneus interface). Proteoglycan depletion was determined by measuring the safranin $\mathrm{O}^{+}$area and results were normalized for the length of the bone covered by cartilage.

Bone-marrow transplantation, myeloid progenitors, leukocytes and SMCs. Transplantation of retrovirally transduced bone-marrow and colonyforming unit (c.f.u.) assays were performed as described ${ }^{46}$. For FACS analysis, blood samples $(\sim 1 \mathrm{ml})$ were stained with primary antibodies for $30 \mathrm{~min}$ at $4{ }^{\circ} \mathrm{C}$. Aspecific interaction of antibodies with the Fc receptor was avoided by incubation with anti-mouse Fcyll//II receptor (Pharmingen, San Diego, California) before staining. Data were analyzed with CellQuest software (Beckton Dickinson, San Jose, California). To determine the effect of antiFlt1 on monocyte/macrophage activity, peritoneal leukocytes ( $78 \%$ monocytes) were collected $72 \mathrm{~h}$ after injection of thioglycollate. $24 \mathrm{~h}$ after plating, cells were incubated with saline, anti-Flt1 $(50 \mu \mathrm{g} / \mathrm{ml})$, rhPIGF-2 $(100 \mathrm{ng} / \mathrm{ml})$ or a combination of rhPIGF-2 $(100 \mathrm{ng} / \mathrm{ml})$ plus anti-Flt 1 $(50 \mu \mathrm{g} / \mathrm{ml})$. Another $24 \mathrm{~h}$ later, MCP-1 and TNF- $\alpha$ levels were determined by ELISA. Adhesion of bone marrow-derived neutrophils was measured by manual counting 30 min after plating in the presence of saline or anti-Flt1. For isolation of SMCs, media fragments of the aorta were incubated for $16 \mathrm{~h}$ at $37^{\circ} \mathrm{C}$ in DMEM containing $0.15 \%$ collagenase, 5\% FCS and antibiotics. After incubation, SMCs were sedimented by centrifugation $(400 \mathrm{~g}$ $10 \mathrm{~min}$ ), resuspended in DMEM $+10 \% \mathrm{FCS}$ and grown at $37^{\circ} \mathrm{C}$ in a humidified atmosphere of $5 \% \mathrm{CO}_{2}$ in air. Cells were routinely used from the third to the sixth passage. Proliferation of SMCs was studied as described for ECs, using growth factors at $100 \mathrm{ng} / \mathrm{ml}$ (ref. 11).

Adenoviral PIGF gene transfer in the ear. Adenovirus expressing PIGF was generated by cloning the murine PIGF- 2 cDNA into the PAC-CMVpLPA vector. $5 \times 10^{7}$ p.f.u. of adenovirus in $5 \mu \mathrm{IBS} / 3 \%$ glycerol was injected into the ear skin of 4-6-wk-old female nude mice ${ }^{19}$. For macroscopy, ears were flat mounted in immersion oil on glass slides and coverslipped. Ears were viewed with a Wild M400 Photomacroscope and images were captured using a SPOT Insight Digital camera. For histology, ears were fixed and processed for $1 \mu \mathrm{m}$ Giemsa-stained Epon sections as described ${ }^{20}$.
Mouse model of myocardial and limb ischemia. For therapeutic angiogenesis, a subcutaneously implanted osmotic minipump (Alzet, type 2001, Iffa Credo, Belgium) continuously delivered during $7 \mathrm{~d}$ a daily dose of $1.5 \mu \mathrm{g}$ of hVEGF $_{165}$, hVEGF $_{121}$, mPIGF-2 (all from R\&D Systems), hPIGF-1 (Geymonat SpA, Anagni, Italy) or hPIGF-2 (ReliaTech) (in quantities expressed as active dimer). Thereafter, infarcted hearts were processed for morphometric analysis after immunostaining for endothelial thrombomodulin (all vessels) or for smooth muscle $\alpha$-actin (mature SMC-covered vessels). To induce limb ischemia, unilateral right or bilateral ligations of the femoral artery and vein (proximal to the popliteal artery) and the cutaneous vessels branching from the caudal femoral artery side branch were performed without damaging the nervus femoralis. rhPIGF-2 and rhVEGF were administered as described above. Two superficial preexisting collateral arterioles, connecting the femoral and saphenous artery, were used for analysis. Functional perfusion measurements of the collateral region were performed using a Lisca PIM II camera (Gambro, Breda, the Netherlands) and analyzed as described ${ }^{47}$. Perfusion, averaged from 3 images per mouse in the upper hindlimb (adductor region where collaterals enlarge) or in total hind limb, was expressed as a ratio of right (ischemic) to left (normal) limb. Spontaneous mobility was scored by monitoring the gait abnormalities, the position of right foot in rest and after manipulation, and the 'tailabduction-reflex'. Mice were scored 0 when one observation was abnormal and 1 when normal. An endurance exercise swim test for mice was developed. Mice were conditioned for $9 \mathrm{~d}$ to swim in a $31^{\circ} \mathrm{C}$ controlled swimming pool in non-stressed conditions. At $d 10$, baseline exercise time for each mouse was determined using a counter-current swimming pool (31 ${ }^{\circ} \mathrm{C}$; flow at $\left.0.2 \mathrm{~m} / \mathrm{s}\right)^{48}$. For determining maximal endurance exercise, the total swimming period until fatigue, defined as the failure to rise to the surface of the water to breathe within $7 \mathrm{~s}$, was assessed. At $d 11$, the femoral artery was occluded, and at $d 18$, minipumps were removed under isoflurane anesthesia before endurance exercise. Recovery of functionality was expressed as a ratio to the baseline exercise time. Fluorescent microspheres (yellow-green, $15 \mu \mathrm{m}, 1 \times 10^{6}$ beads per $\mathrm{ml}$, Molecular Probes, Eugene, Oregon) were administered after maximal vasodilation (sodium nitroprusside, $50 \mathrm{ng} / \mathrm{ml}$, Sigma), processed and flow was calculated as described ${ }^{49}$. Bismuth gelatino-angiography was performed as described ${ }^{11}$ and photoangiographs (Nikon D1 digital camera) were analyzed in a blinded manner. Collateral side branches were categorized as follows: second-generation collateral arterioles directly branched off from the main collateral, whereas third-generation collateral arterioles were orientated perpendicularly to the second-generation branches. The number of collateral branches per $\mathrm{cm}$ length of the primary collateral arteriole was counted. Fluoroangiography was performed with a modified version of a described protocol ${ }^{11}$. Images were reconstructed with a Zeiss LSM510 confocal laser microscope. After perfusion-fixation, the 2 superficial collateral arterioles were post-fixed in paraformaldehyde $1 \%$ and paraffin-embedded. $125-\mu \mathrm{m}$ cross-sections per superficial collateral, starting from the midzone and ranging over $1.95 \mathrm{~mm}$ to each end, were morphometrically analyzed. Collateral side branches were categorized as second generation (luminal area $>300 \mu \mathrm{m}^{2}$ ) or third generation $\left(<300 \mu \mathrm{m}^{2}\right)$. Total perfusion area was calculated using the total sum of the side branch luminal areas. Capillary density was determined by immunostaining for thrombomodulin. Wall thickness of fully SMC-covered vessels was morphometrically measured on histological sections, after smooth muscle $\alpha$-actin staining. For all treatment groups, 6 cross-sections (150 $\mu \mathrm{m}$ apart) were analyzed per main collateral. Only second-generation collateral arterioles larger than $300 \mu \mathrm{m}^{2}$ were included in the analysis. At least 10 measurements of wall thickness of the second-generation collateral arterioles were obtained.

Note: Supplementary information is available on the Nature Medicine website.

Acknowledgments

We thank A. Bouché, K. Vandevelde, Y. Wing Man, I. Vanlinthout, M. De Mol, K. Maris, B. Vanwetswinkel, A. Manderveld, B. Hermans, P. Van Wesemael, S. Jansen, W. Martens, A. Vandenhoeck, S. Terclavers, S. Wyns, W. Landuyt and S. Torrekens for assistance. This work was supported in part by the European Union (Biomed BMH4-CT98-3380), Actie Levenslijn (\#7.0019.98), 
FWO (G012500 and G032401) and a KUL/OT grant (TBA/00/27). A.L. and

V.C. are FWO research fellows. M.T. is an IWT research fellow.

\section{Competing interests statement}

The authors declare competing financial interests: see the website (http://medicine.nature.com) for details.

\section{RECEIVED 10 APRIL; ACCEPTED 31 MAY 2002}

1. Ferrara, N. Role of vascular endothelial growth factor in regulation of physiologica angiogenesis. Am. J. Physiol. Cell. Physiol. 280, C1358-C1366 (2001).

2. Post, M.J., Laham, R., Sellke, F.W. \& Simons, M. Therapeutic angiogenesis in cardiology using protein formulations. Cardiovasc Res. 49, 522-531 (2001).

3. Isner, J.M. Myocardial gene therapy. Nature 415, 234-239 (2002).

4. Carmeliet, P. \& Jain, R.K. Angiogenesis in cancer and other diseases. Nature 407, 249-257 (2000).

5. Veikkola, T., Karkkainen, M., Claesson-Welsh, L. \& Alitalo, K. Regulation of angiogenesis via vascular endothelial growth factor receptors. Cancer Res. 60, 203-212 (2000).

6. Hiratsuka, S., Minowa, O., Kuno, J., Noda, T. \& Shibuya, M. Flt-1 lacking the tyrosine kinase domain is sufficient for normal development and angiogenesis in mice. Proc. Natl. Acad. Sci. USA 95, 9349-9354 (1998).

7. Shibuya, M. Structure and function of VEGF/VEGF-receptor system involved in angiogenesis. Cell Struct. Funct. 26, 25-35 (2001).

8. Persico, M.G., Vincenti, V. \& DiPalma, T. Structure, expression and receptor-binding properties of placenta growth factor (PIGF). Curr. Top. Microbiol. Immunol. 237, 31-40 (1999).

9. Fong, G.H., Rossant, J., Gertsenstein, M. \& Breitman, M.L. Role of the Flt-1 receptor tyrosine kinase in regulating the assembly of vascular endothelium. Nature 376, 66-70 (1995).

10. Park, J.E., Chen, H.H., Winer, J., Houck, K.A. \& Ferrara, N. Placenta growth factor Potentiation of vascular endothelial growth factor bioactivity, in vitro and in vivo, and high affinity binding to Flt-1 but not to Flk-1/KDR. J. Biol. Chem. 269, 25646-25654 (1994)

11. Carmeliet, P. et al. Synergism between vascular endothelial growth factor and placental growth factor contributes to angiogenesis and plasma extravasation in pathological conditions. Nature Med. 7, 575-583 (2001).

12. Hiratsuka, S. et al. Involvement of Flt-1 tyrosine kinase (vascular endothelial growth factor receptor-1) in pathological angiogenesis. Cancer Res. 61, 1207-1213 (2001)

13. Weng, D.E. \& Usman, N. Angiozyme: a novel angiogenesis inhibitor. Curr. Oncol. Rep. 3, 141-146 (2001)

14. Ishida, A. et al. Expression of vascular endothelial growth factor receptors in smooth muscle cells. J. Cell. Physiol. 188, 359-368 (2001).

15. Carmeliet, P. Mechanisms of angiogenesis and arteriogenesis. Nature Med. 6 , 389-395 (2000).

16. van Royen, N. et al. Stimulation of arteriogenesis; a new concept for the treatment of arterial occlusive disease. Cardiovasc. Res. 49, 543-553 (2001).

17. Arras, M. et al. Monocyte activation in angiogenesis and collateral growth in the rabbit hindlimb. J. Clin. Invest. 101, 40-50 (1998).

18. Hoefer, I.E., van Royen, N., Buschmann, I.R., Piek, J.J. \& Schaper, W. Time course of arteriogenesis following femoral artery occlusion in the rabbit. Cardiovasc. Res. 49, 609-617 (2001).

19. Pettersson, A. et al. Heterogeneity of the angiogenic response induced in different normal adult tissues by vascular permeability factor/vascular endothelial growth factor. Lab. Invest. 80, 99-115 (2000).

20. Dvorak, H.F., Dvorak, A.M., Manseau, E.J., Wiberg, L. \& Churchill, W.H. Fibrin gel investment associated with line 1 and line 10 solid tumor growth, angiogenesis, and fibroplasia in guinea pigs. Role of cellular immunity, myofibroblasts, microvascular damage, and infarction in line 1 tumor regression. J. Natl. Cancer Inst. 62, 1459-1472 (1979).

21. McLeod, D.S. et al. Localization of VEGF receptor-2 (KDR/Flk-1) and effects of blocking it in oxygen-induced retinopathy. Invest. Ophthalmol. Vis. Sci. 43, 474-482 (2002).

22. Celletti, F.L. et al. Vascular endothelial growth factor enhances atherosclerotic plaque progression. Nature Med. 7, 425-429 (2001).
23. Moulton, K.S. Plaque angiogenesis and atherosclerosis. Curr. Atheroscler. Rep. 3, 225-233 (2001).

24. Lee, D.M. \& Weinblatt, M.E. Rheumatoid arthritis. Lancet 358, 903-911 (2001).

25. Weber, A.J. \& De Bandt, M. Angiogenesis: general mechanisms and implications for rheumatoid arthritis. Joint Bone Spine 67, 366-383 (2000).

26. Miotla, J., Maciewicz, R., Kendrew, J., Feldmann, M. \& Paleolog, E. Treatment with soluble VEGF receptor reduces disease severity in murine collagen-induced arthritis. Lab. Invest. 80, 1195-1205 (2000).

27. Sone, H. et al. Neutralization of vascular endothelial growth factor prevents collagen-induced arthritis and ameliorates established disease in mice. Biochem. Biophys. Res. Commun. 281, 562-568 (2001).

28. Courtenay, J.S., Dallman, M.J., Dayan, A.D., Martin, A. \& Mosedale, B. Immunisation against heterologous type II collagen induces arthritis in mice. Nature 283, 666-668 (1980).

29. Folkman, J. Angiogenesis-dependent diseases. Semin. Oncol. 28, 536-542 (2001).

30. Bottomley, M.J. et al. Placenta growth factor (PIGF) induces vascular endothelial growth factor (VEGF) secretion from mononuclear cells and is co-expressed with VEGF in synovial fluid. Clin. Exp. Immunol. 119, 182-188 (2000).

31. Lyden, D. et al. Impaired recruitment of bone-marrow-derived endothelial and hematopoietic precursor cells blocks tumor angiogenesis and growth. Nature Med. 7, 1194-1201 (2001).

32. Riessen, R., Kearney, M., Lawler, J. \& Isner, J.M. Immunolocalization of thrombospondin-1 in human atherosclerotic and restenotic arteries. Am. Heart J. 135, 357-364 (1998).

33. Carmeliet, P. VEGF gene therapy: stimulating angiogenesis or angioma-genesis? Nature Med. 6, 1102-1103 (2000).

34. Lazarous, D.F. et al. Basic fibroblast growth factor in patients with intermittent claudication: results of a phase I trial. J. Am. Coll. Cardiol. 36, 1239-1244 (2000).

35. Hattori, K. et al. Placental growth factor reconstitutes hematopoiesis by recruiting VEGFR1+ stem cells from bone-marrow microenvironment. Nature Med. 8, advance online publication, 1 July 2002 (doi:10.1038/nm740).

36. Sawano, A. et al. Flt-1, vascular endothelial growth factor receptor 1 , is a novel cell surface marker for the lineage of monocyte-macrophages in humans. Blood 97, 785-791 (2001).

37. Clauss, M. Functions of the VEGF receptor-1 (FLT-1) in the vasculature. Trends Cardiovasc. Med. 8, 241-245 (1998).

38. Brenchley, P.E. Angiogenesis in inflammatory joint disease: a target for therapeutic intervention. Clin. Exp. Immunol. 121, 426-429 (2000).

39. Libby, P. What have we learned about the biology of atherosclerosis? The role of inflammation. Am. J. Cardiol. 88, 3J-6J (2001).

40. Lutgens, E. et al. Both early and delayed anti-CD40L antibody treatment induces a stable plaque phenotype. Proc. Natl. Acad. Sci. USA 97, 7464-7469 (2000)

41. Simons, M. et al. Clinical trials in coronary angiogenesis: issues, problems, consensus: an expert panel summary. Circulation 102, E73-E86 (2000).

42. Witte, L. et al. Monoclonal antibodies targeting the VEGF receptor-2 (Flk1/KDR) as an anti-angiogenic therapeutic strategy. Cancer Metastasis Rev. 17, 155-161 (1998).

43. Luttun, A. et al. Lack of plasminogen activator inhibitor-1 promotes growth and abnormal matrix remodeling of advanced atherosclerotic plaques in apolipoproteindeficient mice. Arterioscler. Thromb. Vasc. Biol. 22, 499-505 (2002).

44. Matthys, P. et al. Enhanced autoimmune arthritis in IFN- $\gamma$ receptor-deficient mice is conditioned by mycobacteria in Freund's adjuvant and by increased expansion of Mac-1+ myeloid cells. J. Immunol. 163, 3503-3510 (1999).

45. Matthys, P. et al. Anti-IL-12 antibody prevents the development and progression of collagen-induced arthritis in IFN- $\gamma$ receptor-deficient mice. Eur. J. Immunol. 28, 2143-2151 (1998).

46. Priller, J. et al. Targeting gene-modified hematopoietic cells to the central nervous system: use of green fluorescent protein uncovers microglial engraftment. Nature Med. 7, 1356-1361 (2001).

47. Couffinhal, T. et al. Mouse model of angiogenesis. Am. J. Pathol. 152, 1667-1679 (1998).

48. Matsumoto, K., Ishihara, K., Tanaka, K., Inoue, K. \& Fushiki, T. An adjustable-current swimming pool for the evaluation of endurance capacity of mice. J. Appl. Physiol. 81, 1843-1849 (1996).

49. Carmeliet, P. et al. Impaired myocardial angiogenesis and ischemic cardiomyopathy in mice lacking the vascular endothelial growth factor isoforms VEGF164 and VEGF188. Nature Med. 5, 495-502 (1999). 

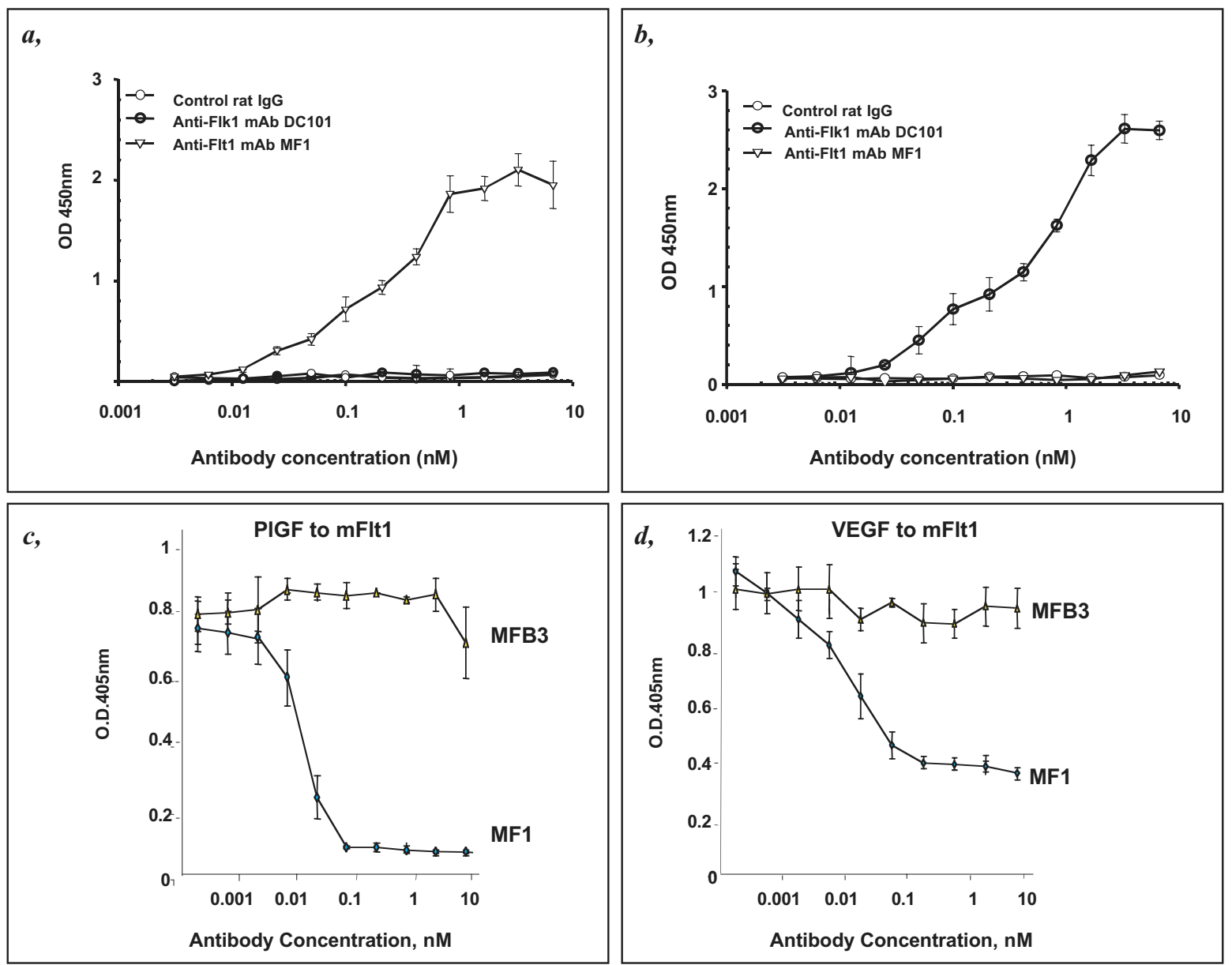

Supplementary Fig. A: Anti-Flt1 specifically blocks binding of VEGF and PIGF to Flt1. a and b, anti-Flt1 (MF1) specifically bound Flt1 (a) but not Flk1 (b), whereas anti-Flk1 (DC101) specifically bound Flk1 (b) but not Flt1 (a). c and d, anti-Flt1 significantly inhibited binding of PIGF (c) and VEGF (d) to FIt1 with IC50 of $0.1 \mathrm{nM}$ and $0.3 \mathrm{nM}$, respectively, whereas a control MFB3 antibody did not interfere with ligand binding. Methods:

Generation of anti-Flt1: Lewis rats (Harlan Sprague-Dawley Inc., Indianapolis, Indiana) were primed with a subcutaneous injection of murine Flt1-Fc (100 mg; extracellular domain of Flt1 fused to Fc-fragment; generated at Imclone) emulsified in complete Freund's adjuvant (Sigma). Rats received 4 booster intraperitoneal injections at 2-3-wk intervals with $100 \mathrm{mg}$ of Flt1-Fc. Rats showing highest titer of blocking antibody in VEGF/Flt1-Fc blocking assays (see below) were boosted intravenously with $50 \mathrm{mg}$ of Flt1-Fc. $5 \mathrm{~d}$ later, splenocytes were harvested and fused to mouse myeloma cells P3-X63-Ag8.653. Generation of hybridomas and subcloning was performed according to standard protocols. Hybridomas secreting anti-Flt1 were selected for binding to soluble Flt1-Fc and negative binding to Fc protein alone in ELISA. The anti-Flt1 was selected for inhibition of Flt1-Fc/ligand binding as described below. The binding kinetics of anti-Flt1 $(\mathrm{Kd}=2 \times 10-6 \mathrm{M})$ were measured using a BIAcore biosensor (Pharmacia Biosensor). Anti-Flt1 was produced by continuous feed fermentation of hybridoma cells in serum-free medium. Anti-Flt1 was purified from conditioned media by a multi-step chromatography process and assessed for purity by SDS-PAGE and immunoreactivity with soluble Flt1 receptor in ELISA. The negative control rat lgG was purchased from Jackson ImmunoResearch Laboratories (West Grove, Pennsylvania). Protein concentration of antibodies was determined using the BCA method. Characterization: To determine whether anti-Flt1 efficiently bound to VEGF-receptor 1 (Flt1), we performed binding assays in Flt1-AP protein-coated plates (Flt1-AP: extracellular domain of Flt1 fused to human secretory alkaline phosphatase (SEAP)). After sequential incubation with various concentrations of anti-Flt1, goat anti-rat lgG-HRP and colorigenic substrate, we quantified binding by reading on a microtiter plate reader at $450 \mathrm{~nm}$. Subsequently, we measured the efficiency of anti-Flt1 to block binding of Flt1 ligands to their receptor by Flt1/VEGF or FIt1/PIGF blocking assays in plates coated with VEGF or PIGF. After sequential incubation with Flt1-AP (that we preincubated with various concentrations of anti-Flt1) and colorigenic substrate, we measured binding by reading at $405 \mathrm{~nm}$. 

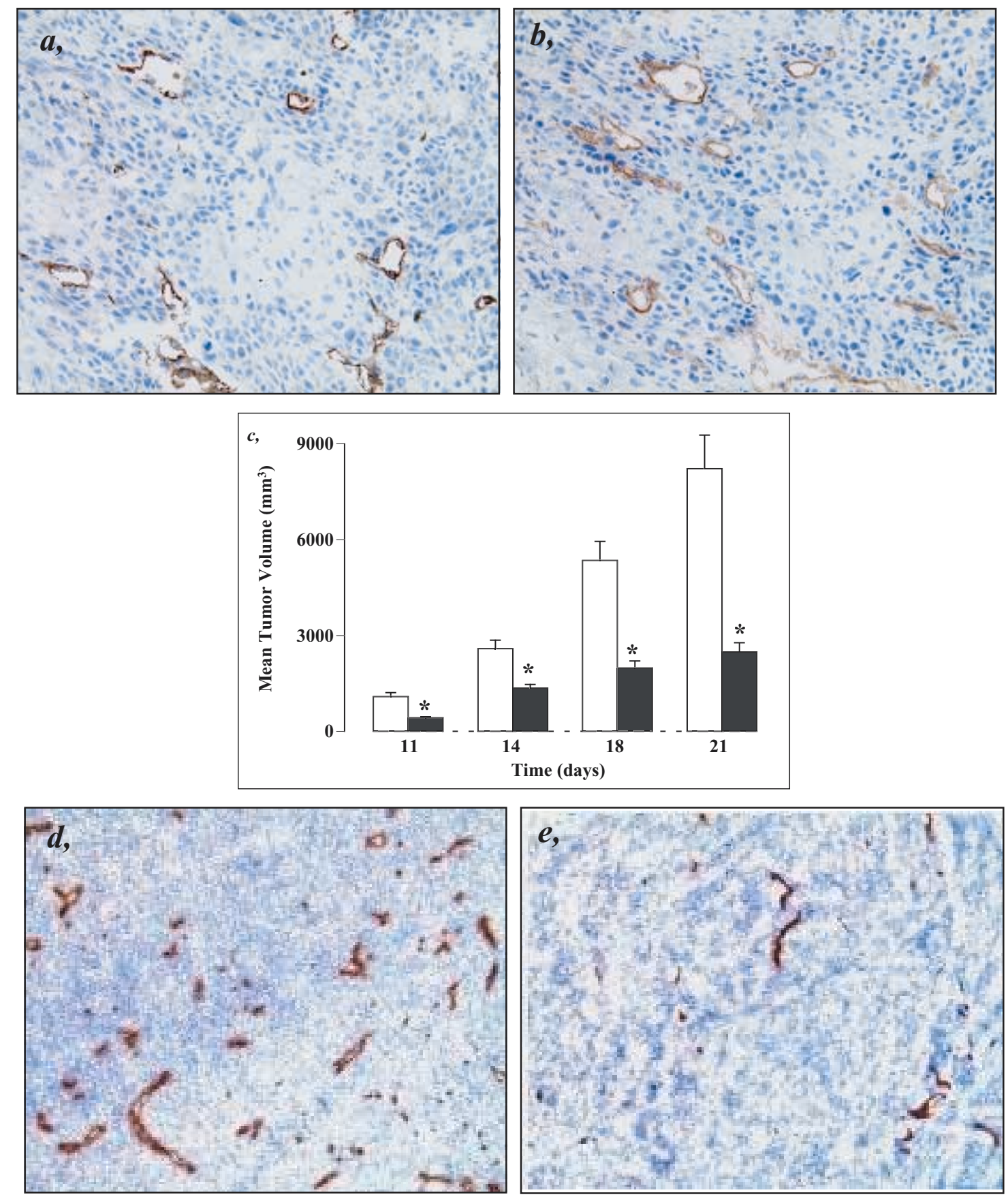

Supplementary Fig. B Flt1 is expressed on epidermoid tumor vessels and anti-Flt1 inhibits growth and vascularization of VEGF or PIGF-overexpressing rat C6 gliomas. Anti-Flt1 in some figure panels is indicated by 'MF1'. a and b, Immunostaining for Flt1 revealing expression of Flt1 on endothelium of vessels in A431 epidermoid tumors, but not on tumor cells a. b, Shows an adjacent CD31 stained section. c, Graph showing volumes of PIGF-overexpressing tumors (mean \pm s.e.m., $\mathrm{n}=12$ ) of mice treated with PBS (white) or anti-Flt1 (black). After 3 wk, anti-Flt1 treatment reduced tumor volume 4-fold as compared with control mice. Anti-Flt also reduced volumes of VEGF-overexpressing rat C6 gliomas (data not shown). $d$ and e, CD31 staining revealing reduced vessel density in PIGF-overexpressing tumors grown in anti-Flt1-treated mice (e) as compared with control mice (d). In addition, vessels were significantly smaller in anti-Flt1-treated mice. Similar results were obtained with VEGFoverexpressing tumors (data not shown). Methods: VEGF or PIGF-transduced rat C6 gliomas were established by injecting the tumor cells into athymic nude mice subcutaneously in the right flank. $3 \mathrm{~d}$ after tumor engraftment, randomized groups of mice received intraperitoneal injection of anti-Flt1 at a dose of $1 \mathrm{mg}$ every $3 \mathrm{~d}$ or PBS. 

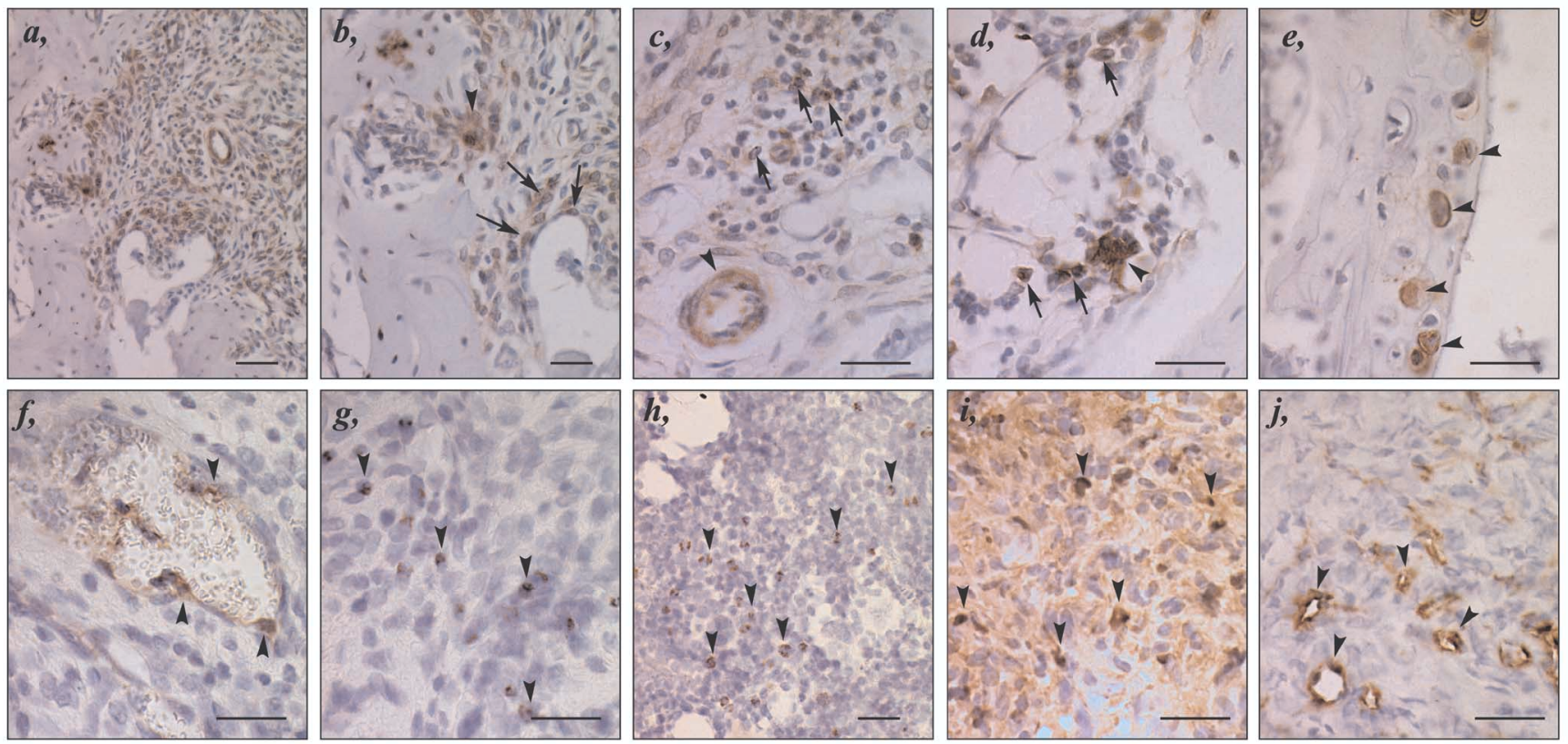

Supplementary Fig. C: Expression of Flt1, Flk1, PIGF and VEGF in inflamed arthritic joints. a-e, Immunostaining revealing that VEGF was highly expressed in rheumatoid synovium (a). VEGF was detected on spindle-shaped cells (likely synoviocytes; arrows in b) and multinucleated cells (likely osteoclasts; arrowhead in b) at the pannus-bone interface. In addition, VEGF was present on endothelial cells lining synovial neovessels (arrowhead in c) and on inflammatory cell clusters in the vicinity of these vessels (arrows in c). Some cells in the bone marrow expressed VEGF (arrows in d). Note the strong expression in a multinucleated osteoclast (arrowhead in d). Finally, VEGF was strongly expressed on chondrocytes (arrowheads in e). $\mathrm{f}-\mathrm{h}$, PIGF was also expressed in arthritic synovium, in particular on endothelial cells of synovial neovessels (arrowheads in $\mathrm{f}$ ), some cells in the inflammatory infiltrate (arrowheads in $\mathrm{g}$ ) and in the bone marrow (arrowheads in h). i and j, Flt1 was expressed on inflammatory cells (arrowheads in i) in the inflamed synovium and on synovial vessels, whereas Flk1 expression was restricted to synovial neovessels (arrowheads in j). Scale bars, $50 \mathrm{~mm}$ in (a), $25 \mathrm{~mm}$ in $(b-j)$. 


\section{ADDENDA}

- Cover page Nature Medicine related to this paper

- News and Views commenting on this paper 


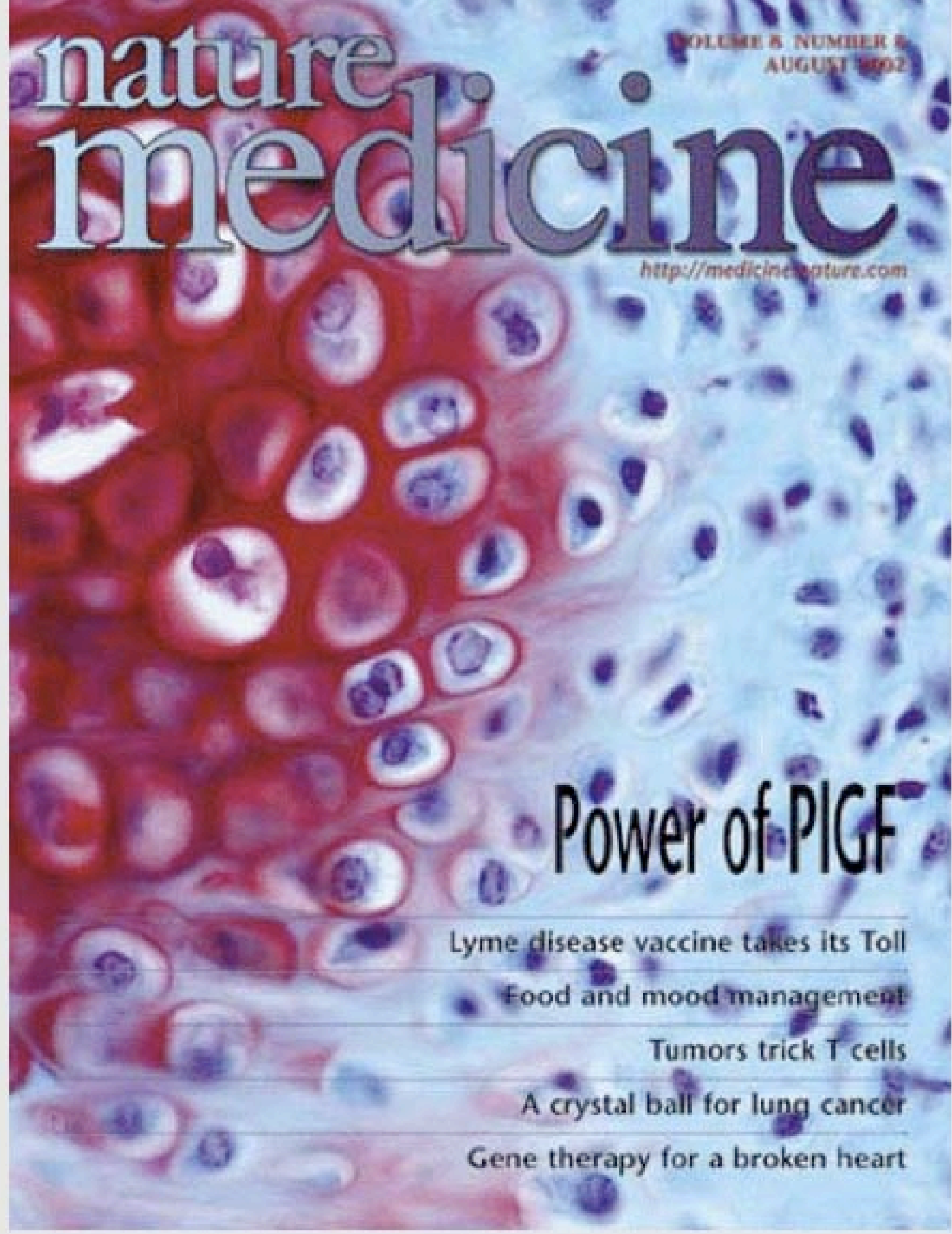

The efficacy and low toxicity of the angiogenic factor placental growth factor (PIGF) and its receptor VEGFR1 may make them preferable to VEGF and its primary receptor VEGFR2 as stimulators of anglogenesis and hematopoiesis. In one of two studles of PIGF's action in the current issue, Hattorl et al. (page 841) demonstrate that PIGF enhances hematopolesis in mice following irradiation-induced bone-marrow suppression. Luttun et al. (page 831) report that delivery of PIGF improved revascularization of heart and limbs in ischemic mice, whereas an antibody against VEGFR1 reduced atherosclerosis, tumor growth and arthritic joint destruction. The cover image illustrates a cross-section of an arthritic joint, with chondrocytes embedded in a red safranin 0-stained matrix of proteoglycans. Magnification, x63 


\title{
VEGF receptor 1 stimulates stem-cell recruitment and new hope for angiogenesis therapies
}

\author{
Efforts to control vessel growth have focused on vascular endothelial growth factor (VEGF) and its primary receptor \\ VEGFR-2. New data could shift that focus to other members of the VEGF family and the receptor VEGFR-1, \\ particularly in inflammatory diseases (pages 831-840 \& 841-849).
}

A drop in tissue blood supply as a consequence of heart attack or stroke is the most common cause of death in the Western world. In addition, millions of patients suffer from chronic ischemia due to insufficient blood supply to tissues, particularly in their legs. The growing population of diabetics is particularly prone to developing these life-threatening conditions. Recent studies have suggested that such vascular complications may be alleviated through the generation of new vessels by angiogenesis, the sprouting of new capillaries from the vascular tree, or arteriogenesis, the remodeling of arteries. However, new vessel growth is not always desirable; aberrant angiogenesis occurs in disorders such as cancer and diabetic retinopathy, and in many types of inflammatory conditions, such as rheumatoid arthritis. Thus, under certain conditions new blood vessel formation may help prevent disease, whereas under others, halting vessel development is a therapeutic goal ${ }^{1}$.

The main regulator of blood vessel growth is vascular endothelial growth factor (VEGF) ${ }^{2}$. VEGF is a small, secreted glycoprotein whose production is increased by tissue hypoxia, and which stimulates the growth and invasion of blood vessels into ischemic tissues. VEGF and its major receptor, VEGFR-2 (also known as FlK-1 Or

\section{UlF ERIKSSON $^{1} \&$ KaRi Alitalo ${ }^{2}$}

fects. These include increased vessel permeability, which leads to tissue edema, fibrin deposition and formation of abnormal vessel structures commonly seen in benign vascular tumors called hemangiomas $^{4}$. Furthermore, only a fraction of the de novo VEGF-generated vessels become functional and stable.

Three recent studies open the door to refining the approaches to promote vessel growth. Two reports in this issue $e^{5,6}$ and one in the 27 June issue of Nature implicate growth factors binding to the other VEGF receptor, VEGFR-1 (Flt-1), as important mediators of stem-cell recruitment and mobilization, angiogenesis and inflammation. Although untested in the clinic, these reports suggest that treatment based on placental growth factor (PIGF) and its receptor VEGFR-1 may in some instances prove more effective, and produce fewer side-effects than application of VEGF itself.

VEGF is a member of a family of growth factors that includes PIGF, VEGF-B, VEGF-C and VEGF-D (ref. 8). VEGF-C and VEGF-D seem to have major roles in lymphangiogenesis and metastatic

or KDR), have been the focus of efforts to develop pro- and anti-angiogenic therapies ${ }^{3}$ Inhibition of the VEGF-VEGFR-2 signal transduction pathway has provided promising antiangiogenic results in preclinical studies. But the use of VEGF to promote vessel growth has resulted in several side ef- spread of tumors via the lymphatic vessels, whereas the roles of PlGF and VEGF-B have remained enigmatic because their deletion from mice does not result in overt abnormalities ${ }^{9,10}$. However, a recent study found that the adult tissues of PlGF-deficient mice display inhibited pathological angiogene- 
sis and arteriogenesis ${ }^{10}$.

VEGF signals via two receptor tyrosine kinases, VEGFR-1 and VEGFR-2. VEGFR-2 has strong intrinsic tyrosine kinase activity, and is the major mediator of endothelial cell proliferation in response to VEGF (ref. 11). VEGFR-1 also serves as a receptor for both PlGF and VEGF-B (ref. 12). Experiments on VEGFR-1 have provided tantalizing data, but have revealed little about its function in adult mice. VEGFR-1-deficient mice die as embryos with excess endothelial cells in the vasculature ${ }^{13}$. Mice deficient in only the tyrosine kinase domain are viable and show no vascular phenotype, but exhibit a defect in monocyte migration in vitro ${ }^{14}$. VEGFR-1 is expressed by endothelial cells but it is also present on inflammatory cells such as monocytes and macrophages. Overall, the precise function of VEGFR-1 has remained about as unclear as that of its ligands PlGF and VEGF-B.

Two reports in this issue address the roles of VEGFR-1 and PlGF in several mouse experimental and disease models. Luttun et $a l .{ }^{5}$ analyzed the role of PIGF in the stimulation of angiogenesis and development of collateral vessels, which serve to bypass an artery obstructed by atherosclerotic lesions in the ischemic heart or limb. Luttun et al. applied PlGF systemically via an osmotic minipump and saw enhancement of angiogenesis in the ischemic myocardium and the growth of collateral side branches in the ischemic limb (Fig. 1). The efficiency of PlGF rivaled that of VEGF, suggesting an important role for VEGFR-1 in angiogenesis and arteriogenesis. PlGF treatment also markedly increased tissue perfusion and improved tissue function. The potent effect of PlGF on the formation of new vessels occurred in part because of a coincidental stimulation of endothelial and vascular smooth-muscle cell growth.

The authors went on to show that blocking monoclonal antibodies against VEGFR-1 suppressed blood-vessel formation in tumors and in ischemic retinas. The antibodies also strongly inhibited atherosclerotic plaque growth as well as autoimmune arthritis, including inflammation and joint destruction (Fig. 1). By comparison, blocking antibodies against VEGFR-2 were somewhat more effective against tumor growth than anti-VEGFR-1, but had no effect on inflammation. The anti-inflammatory effect of VEGFR-1 antibodies occurred as a result of reduced mobilization of bone marrow-derived hematopoietic stem/precursor cells into the peripheral blood, and reduced activation and tissue infiltration of VEGFR1-expressing leukocytes.

The study by Hattori et al. ${ }^{6}$ investigated the mechanism underlying VEGFR-1-dependent recruitment of hematopoietic cells. This study builds on the authors previous observation that VEGFR-2+ bone marrow-derived cells failed to engraft and repopulate lethally irradiated mice. The new work was also prompted by findings that Drosophila VEGF supports the motility of hemocytes, the ancestral fly counterparts of the mammalian hematopoietic cells ${ }^{15}$.

The authors used a model of hematopoietic cell recovery which involved transient suppression of mouse bone marrow by depletion of cycling cells with the toxic nucleoside analog, 5-fluorouracil. They then investigated the role of the VEGFR-1 signals during the hematopoietic recovery. Treatment with a blocking monoclonal antibody to VEGFR-1, but not with an antibody to VEGFR-2, inhibited the hematopoietic recovery. In contrast, delivery of PlGF via an adenovirus improved it. Detailed analysis showed that PlGF treatment directly increased VEGFR-1 ${ }^{+}$ hematopoietic stem cell recruitment and mobilization (Fig. 1). In addition, PIGF treatment indirectly stimulated a later phase of hematopoietic recovery by inducing matrix metalloproteinase9 (MMP-9) mediated release of soluble Kit ligand. The Kit ligand then induced the translocation of the resting hematopoietic stem cells in the bone marrow to a microenvironment conducive to stem cell proliferation and differentiation.

In yet a third development within the same theme, Gerber et al. ${ }^{7}$ show that VEGF regulates hematopoietic stem-cell survival by an apparent autocrine loop mechanism. The authors arrived at this conclusion in experiments where they deleted the VEGF gene selectively from hematopoietic cells using a conditional gene-targeting method. The VEGF-deleted hematopoietic stem cells failed to survive or differentiate in cell culture and failed to repopulate the hematopoietic compartment of lethally irradiated mice, de- spite properly homing to the bone marrow. The requirement for VEGF seemed to be cell intrinsic, as capture of extracellular VEGF by soluble VEGFR-1 could not mimic VEGF gene ablation in the wild-type hematopoietic stem cells. On the other hand, ligands specific for either VEGFR-1 or VEGFR-2 could rescue the gene-deleted hematopoietic stem cells, and restored their survival and colony formation in in vitro culture assays. In light of these findings and others $^{16}$, the role of VEGFR-2 in hematopoietic and endothelial stemcell recruitment and mobilization clearly warrants further study.

Although the findings that PlGF has in vivo angiogenic activity are not completely unprecendented ${ }^{17}$, the new results are surprising in view of the low potency of PlGF and other tested VEGFR-1 ligands in endothelial-cell proliferation and angiogenesis assays in vitro $^{2}$. The new data also suggest that although VEGFR-2 may be the driving force of angiogenesis in endothelial cells, much of the biological function of the VEGFR-1 ligand system may lie in the regulation of hematopoietic and inflammatory cells. These results also underscore the recent discovery of the importance of inflammatory cells and MMP-9 in tumor angiogenesis ${ }^{18}$. An intimate relationship between VEGFR-1 and MMP-9 induction has been recently observed also in a lung metastasis model in tumor-bearing mice (M. Shibuya, pers. comm.).

The three studies discussed here also have important clinical implications. They clearly suggest that VEGFR-1 and its specific ligands may provide tools for the therapeutic modulation of angiogenesis, collateral growth, hematopoiesis, atherosclerosis and inflammation. The efficacy and low toxicity profile of PlGF suggest that it might be preferred over VEGF in attempts to stimulate angiogenesis and collateral vessel development for the treatment of ischemic conditions. VEGF-B presumably has equally high therapeutic potential.

VEGFR-1 now stands out as a potential target in attempts to reduce inflammation and atherosclerotic plaque growth by preventing the recruitment and activation of inflammatory cells. The application of VEGFR-1 ligands for hematopoietic stem-cell recruitment and mobilization could be useful in patients with suppressed bone marrow function 
after irradiation and chemotherapy. VEGFR-1 ligand-activated hematopoietic stem cells could be used for stem-cell transplantation or organ restoration. However, problems could arise with systemic VEGFR-1 therapies. In some patients with diabetes, atherosclerosis and tissue ischemia, VEGFR-1 stimulation of neovascularization could also accelerate retinopathy, plaque formation or possibly tumor growth. Thus, a very locoregional form of the therapy would be preferable in such instances. On the other hand, systemic use of VEGFR-1 inhibitors to inhibit tumor angiogenesis could pose problems in patients with compromised hematopoiesis and it could inhibit collateral vessel development in patients with cardiovascular diseases. Thus, more extensive studies of these molecules in experimental disease models are needed before these findings translate into clinical trials. However, this trio of studies will clearly revive and boost the attempts to design therapies based on angiogenesis research.

1. Carmeliet, P. \& Jain, R.K. Angiogenesis in cancer and other diseases. Nature 407, 249-257 (2000).

2. Ferrara, N. \& Davis-Smyth, T. The biology of vascular endothelial growth factor. Endocrine Rev. 18, 4-25 (1997)

3. Ferrara, N. \& Alitalo, K. Clinical applications of angiogenic growth factors and their inhibitors. Nature Med. 5, 1359-1364 (1999).

4. Epstein, S.E., Kornowski, R., Fuchs, S. \& Dvorak, H.F. Angiogenesis therapy: Amidst the hype, the neglected potential for serious side effects. Circulation 104, 115-119 (2001).

5. Luttun, A. et al. Revascularization of ischemic tissues by PIGF treatment and inhibition of tumor angiogenesis, arthritis and atherosclerosis by antiFlt-1 antibody. Nature Med. 8, 831-840 (2002).

6. Hattori, K. et al. Placental growth factor reconstitutes hematopoiesis by recruiting VEGFR $1^{+}$stem cells from bone marrow microenviroment. Nature Med. 8, 841-849 (2002).

7. Gerber, H.-P. et al. VEGF regulates hematopoietic stem cell survival by an internal autocrine loop mechanism. Nature 417, 954-958 (2002)

8. Eriksson, U. \& Alitalo, K. Structure, expression and receptor-binding properties of novel vascular endothelial growth factors. Curr. Top. Microbiol. Immunol. 237, 41-57 (1999).

9. Aase, K. et al. Vascular endothelial growth factorB-deficient mice display an atrial conduction defect. Circulation 104, 358-364 (2001).

10. Carmeliet, P. et al. Synergism between vascular endothelial growth factor and placental growth factor contributes to angiogenesis and plasma extravasation in pathological conditions. Nature Med. 7, 575-583 (2001).

11. Matsumoto, T. \& Claesson-Welsh, L. VEGF receptor signal transduction. SCi. STKE 2001, RE21 (2001).
12. Shibuya, M. Structure and dual function of vascular endothelial growth factor receptor-1 (Flt-1). Int. J. Biochem. Cell Biol. 33, 409-420 (2001).

13. Fong, G.H., Zhang, L., Bryce, D.M. \& Peng, J. Increased hemangioblast commitment, not vascular disorganization, is the primary defect in flt-1 knock-out mice. Development 126, 3015-3025 (1999).

14. Hiratsuka, S., Minowa, O., Kuno, J., Noda, T. \& Shibuya, M. Flt-1 lacking the tyrosine kinase domain is sufficient for normal development and angiogenesis In mice. Proc. Natl. Acad. Sci. USA 95, 9349-9354 (1998).

15. Cho, N.K. et al. Developmental control of blood cell migration by the Drosophila VEGF pathway. Cell 108, 865-876 (2002).

16. Ziegler, B.L. et al. KDR receptor: A key marker defining hematopoietic stem cells. Science $\mathbf{2 8 5}$ 1553-1558 (1999).

17. Ziche, M. et al. Placenta growth factor- 1 is chemotactic, mitogenic, and angiogenic. Lab Invest. 76, 517-531 (1997).

18. Bergers, G. et al. Matrix metalloproteinase-9 triggers the angiogenic switch during carcinogenesis. Nature Cell Biol. 2, 737-744 (2000).

${ }^{1}$ Ludwig Institute for Cancer Research, Stockholm Branch, Stockholm, Sweden ${ }^{2}$ Molecular/Cancer Biology Laboratory and Ludwig Institute for Cancer Research,

Haartman Institute and Biomedicum Helsinki, University of Helsinki, Finland

Email: kari.alitalo@helsinki.fi

\title{
Two-shot cocktail: Adenosine, dopamine and a twist of $\beta \gamma$
}

\author{
The neurotransmitter dopamine underlies most addictive processes-but its faint response to ethanol has \\ puzzled researchers. New research suggests that ethanol interacts synergistically with adenosine and \\ dopamine signaling to amplify the effect of drinking.
}

$\mathrm{T}$ he compulsive abuse of alcohol and other drugs often continues in the face of health, social and financial problems caused by addiction. The tremendous societal impact of alcoholism must be rooted in fundamental neurobiological mechanisms and must reflect a 'rewiring' of the neurocircuitry by chronic drug ingestion. Although this view is at least superficially attractive, clear descriptions of the relevant brain circuitry and the effects of alcohol have been slow to emerge. All drugs of abuse activate brain dopaminergic systems and this activation of 'reward' pathways may initiate the pleasurable effects of the drugs. But the simple idea of chemical reward does not explain why a drug such as nicotine, usually not described as pleasurable on first use, is at least as addictive as cocaine, a drug that produces strong initial rewarding effects.

A more attractive incarnation of the 'dopamine activation' hypothesis holds that dopamine systems are critical not just for inducing reward, but

\section{R. AdRON HARRIS \& RICHARD A. MORRISETT}

for the more complex processes of learning about reward and regulating reward-related behaviors. Thus, dopamine-mediated changes in synaptic function (that is, synaptic plasticity) would persist after termination of the initial reward and 'reward memory' would underlie craving and other longlasting aspects of drug addiction. This outlook suggests that drugs of abuse provide much stronger activation of dopamine pathways than natural reinforcers such as food or sex, and stimulate an abnormal degree of synaptic plasticity ${ }^{1}$. This idea is readily applicable to drugs such as cocaine or methamphetamine, which act directly on dopamine terminals and likely flood the synapse with neurotransmitter. However, ethanol releases dopamine only indirectly by increasing neuronal firing. Thus, the magnitude of dopamine release by ethanol appears to be much less than that produced by stimulants.

For ethanol to be able to 'hijack' the reward memory machinery, as proposed for cocaine, a postsynaptic mechanism to amplify the relatively weak presynaptic actions of ethanol might be required. In a recent report in Cell, Yao, Diamond and colleagues provide evidence for such a mechanism ${ }^{2}$. Their report describes a synergistic postsynaptic interaction between ethanol's effects on synaptic levels of dopamine and another important neuromodulator, adenosine, and thus provides an elegant example for such a postsynaptic amplification-one almost as complex as the disease of alcoholism itself!

Yao et al. used a common immortalized neural culture system, NG-108-15 cells, as a test bed for investigating how ethanol can amplify the actions of a major type of dopamine receptor, the D2 dopamine receptor subtype (Fig. 1). Most investigators agree that ethanol weakly activates dopamine release and 